التصميم الداخلى المستدام للحيزات التكيفية.

\title{
Sustainable Interior Design for Adaptive spaces.
}

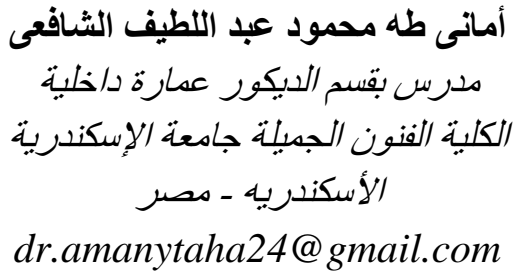

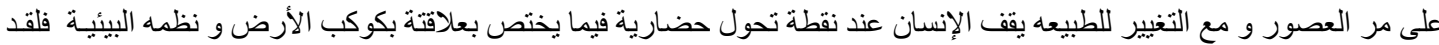

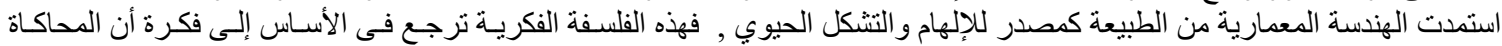

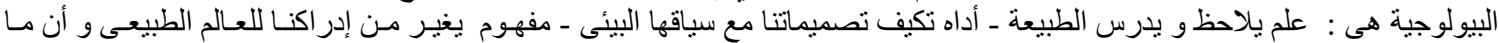

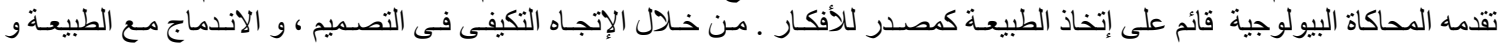

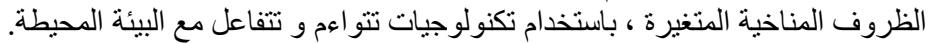

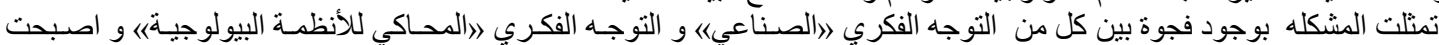

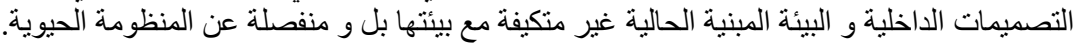

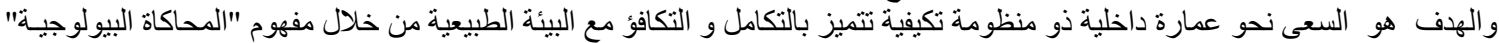

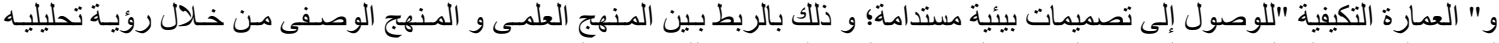

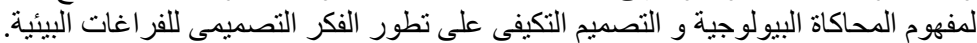

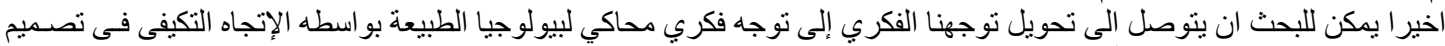

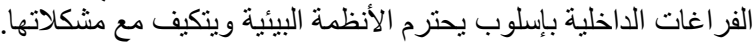
فمبدأ التكيف من أهم المبادئ التيى يجب التبل ان نتعلمها من الطبييعه و نعيد إحيائها و ضرورة العمل على احترام القو انين الحيويه و البيولوجيه

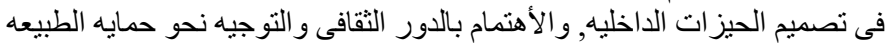
الكلمات المفتاحية:

(المحاكاة البيولوجية" و " العمارة التكيفية " الأستدامة).

\section{Abstract:}

Throughout the ages and with the changes of nature, humanity stands now at a turning point in its relationship with the planet and its ecosystems. Architecture has been derived from nature as a source of inspiration and biological formation. The idea that biological simulation is a science that observes and studies nature as a concept that changes the awareness of biological simulation. Through the adaptive trend in design, integration with nature and climatic changes conditions, using technologies that are compatible with the surrounding environment.

The problem is the gap between the industrial and the simulated biological systems' intellectual approach. The interior design and architecture became not adapted to their environment, but rather separate from the biological systems.

The goal is to create an interior architecture with an adaptive system characterized by integration with the natural environment through the concept of "biological simulation" and "adaptive architecture" to reach sustainable designs. That is by linking the scientific method and the descriptive approach through an analytical view of the concept of biological simulation and adaptive design development.

Finally, the research can reach the transformation of the intellectual approach into the simulation of the natural adaptive approach in interior design with attention to the cultural role and guidance towards protecting and respecting the environmental systems.

Key Words:

(Biological simulation - Adaptive architecture- Sustainable). 


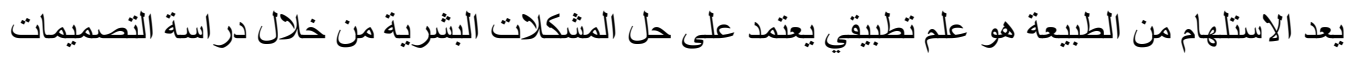

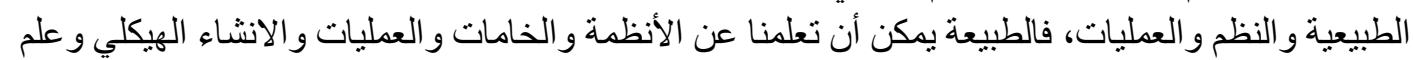

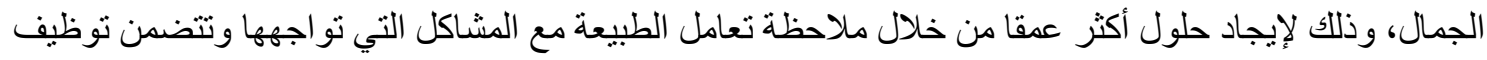

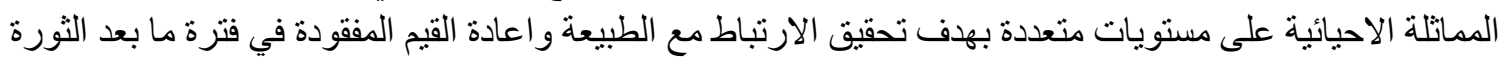

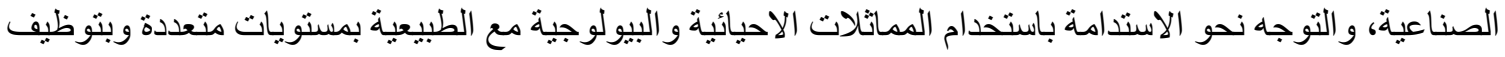

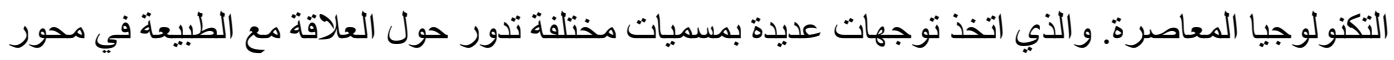

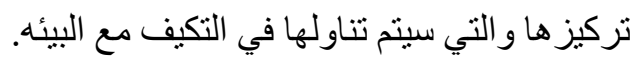

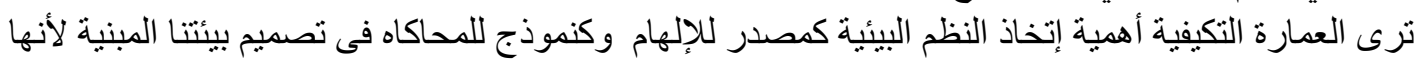

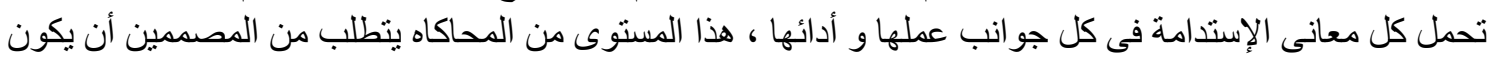

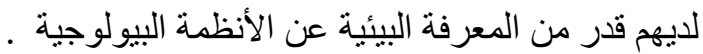

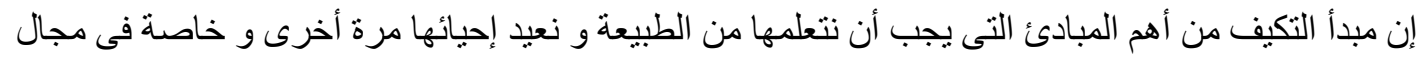

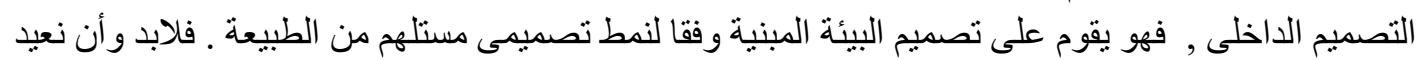

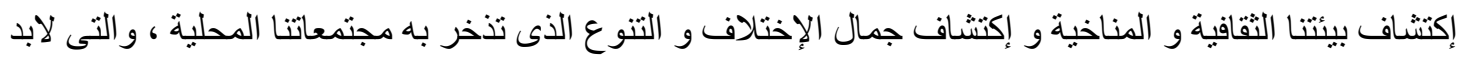

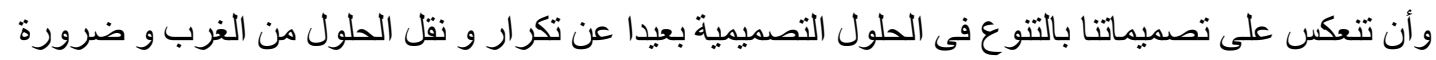

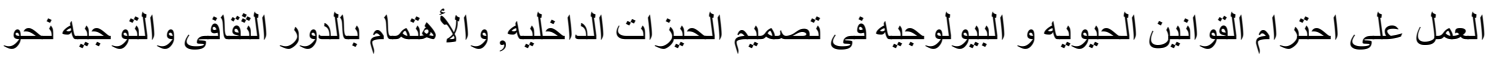

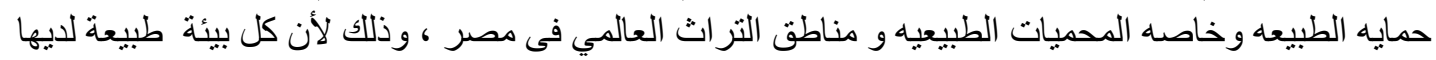

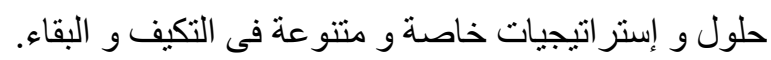

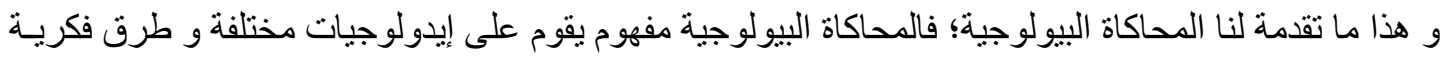

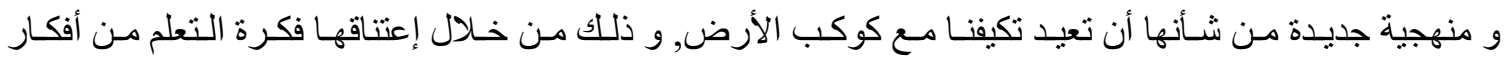

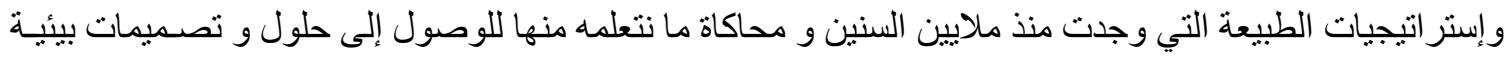

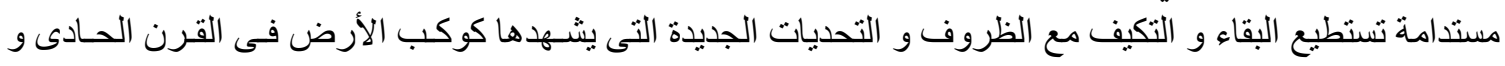

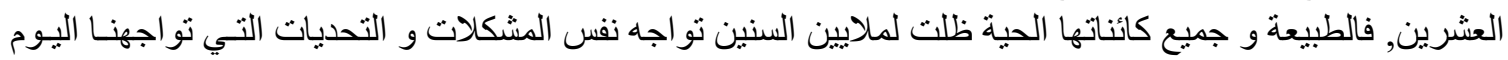

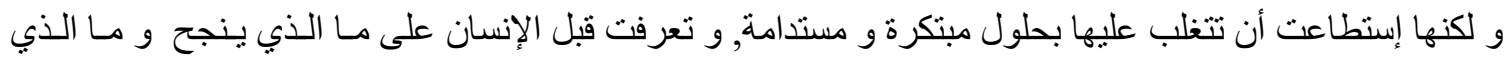

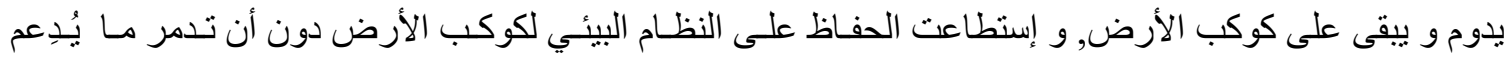

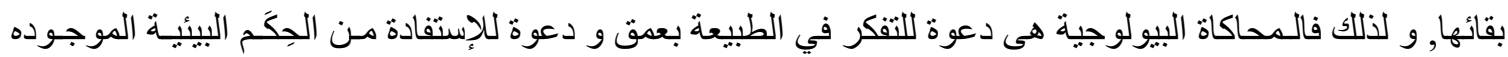

حولنا للوصول إلى تصميمات بيئية مستدامة.

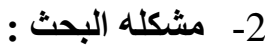

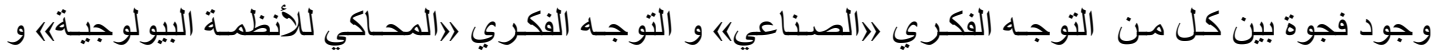

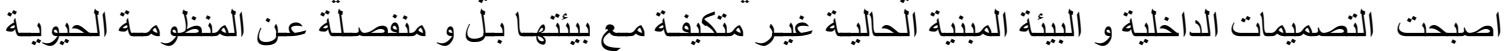

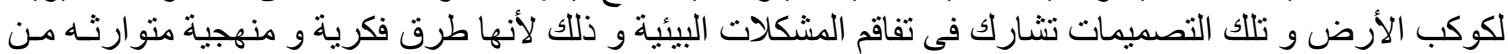

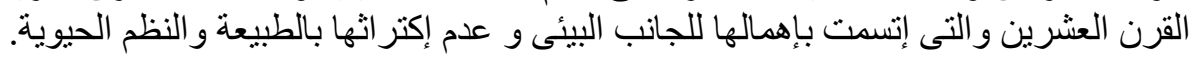

$$
\text { 3- }
$$

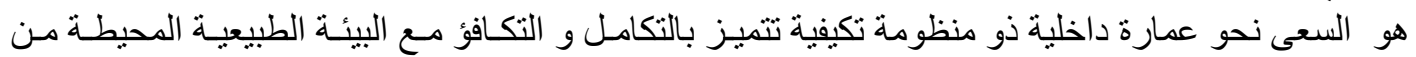

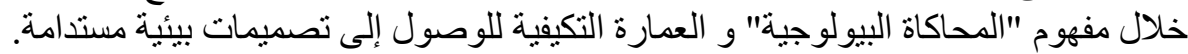

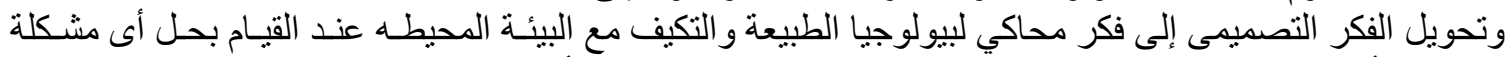

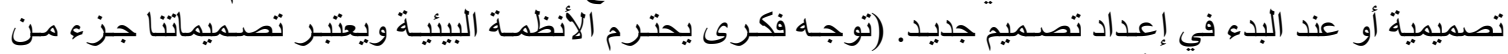

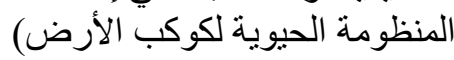

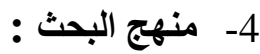

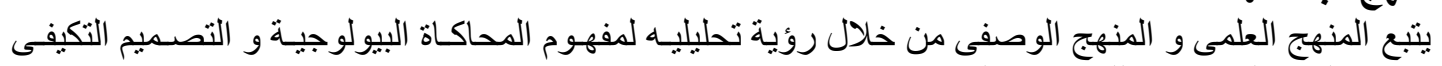
على تطور الفكر التصميمى للفر اغات البيئية ـ دايجرام(1) خلئ رولية 


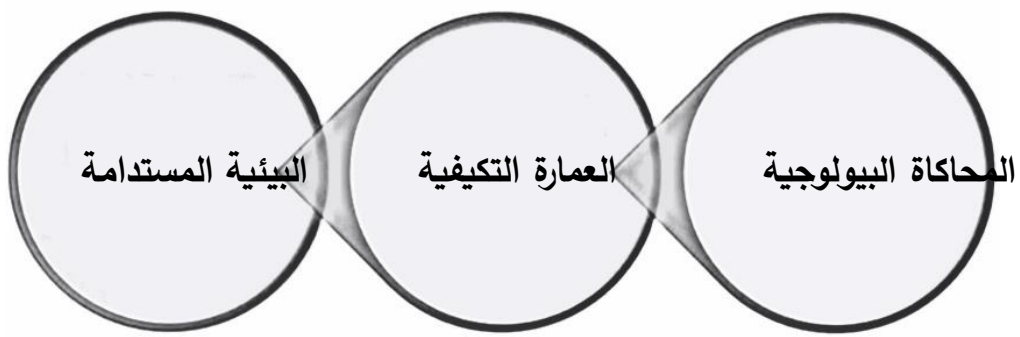

دايجر ام(1) يوضح كيفيه تحقيق الأستدامه من خلال المحاكاة البيلوجية للعمارة التكيفيه

اولا : فكر الإستدامة و فلسفتها في التصميم التكيفي للمحاكاة البيوبوجية:

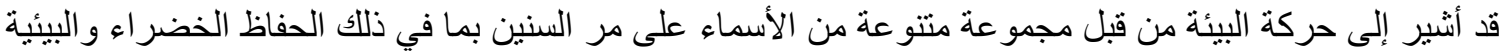

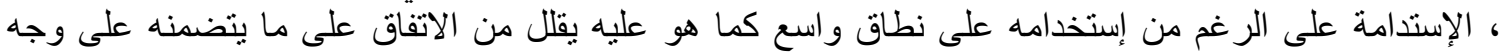

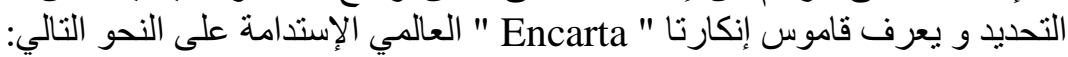
قادرة على البقاء و الإستمر ارية دارية

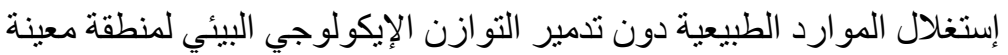

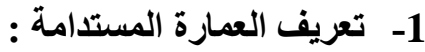

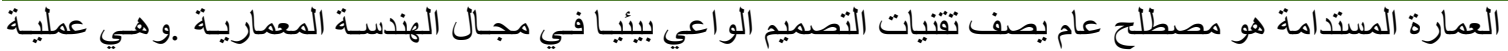

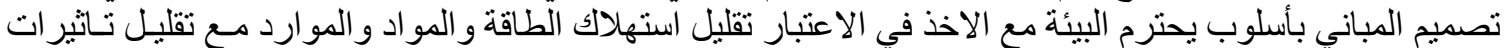

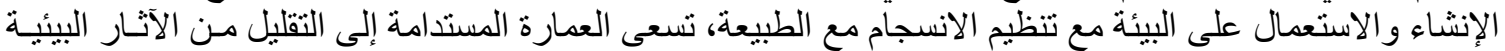

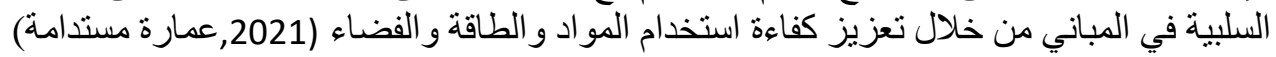

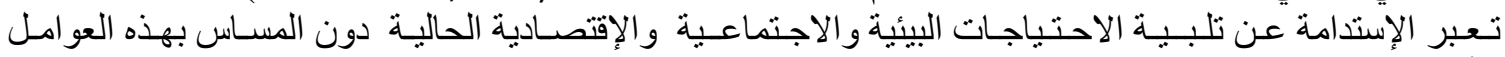
للأجيال المقبلة (Glossary of sustainability terms,2021)

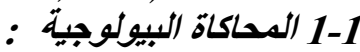
" المقصود بالمحاكاة البيولوجية هي واحده من بين الكثير من الأدو ات التي سُُساعدنا في أن نتعلم كيف نعيش بطريقه

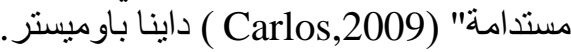

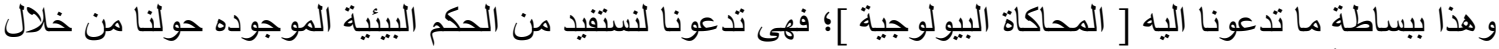

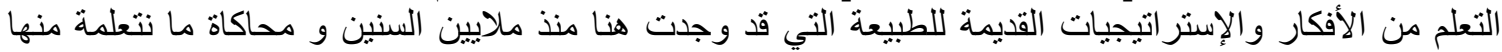

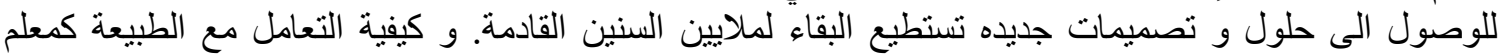
لإثر اء مجال العمارة الداخلية من خلال مفهوم المحاكاة البيولوجية.

$$
\text { BI - O - MIM - IC - RY }
$$

حرفياً, تأتى كلمة BIOMIMICRY من كلمات يونانية ؛[Bios] تعنى [ حياة ] و [Mimesis] تعنى [ محاكاة ]

(Janine M.,1997)

إذاً, فكلمة Biomimicry تعنى [ محاكاة الحياة ] - [ [ محاكاة الطبيعة]

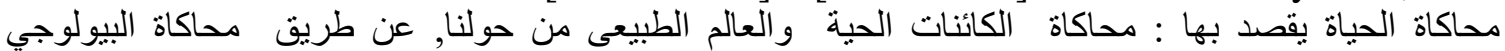

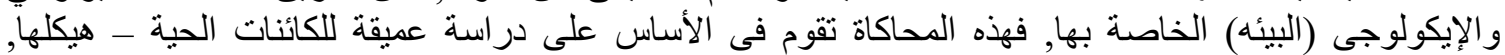

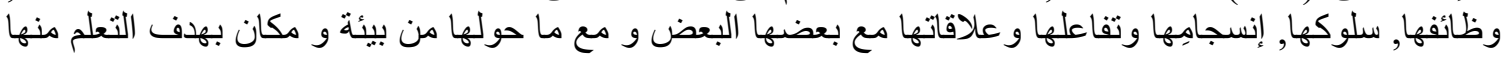

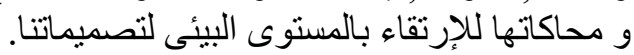

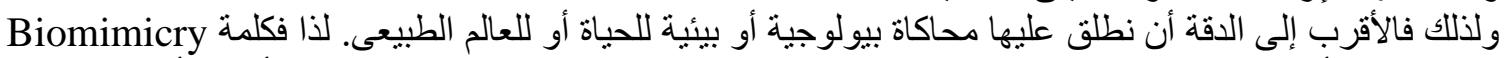

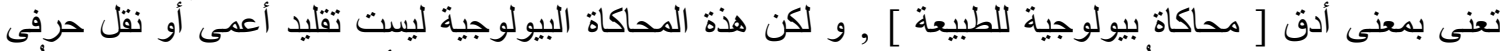

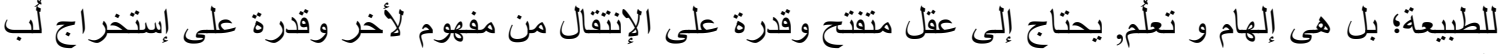
(Vincent,2021). الفكرة 
" فإذا أردت الطير ان فإنك لن تقلد الطير, ولكنك ستقلد إستخدام الأجنحة ورقائقها الهو ائية " جوليان فينسنت

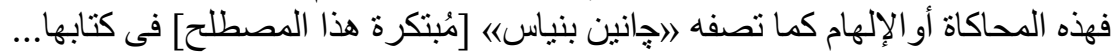

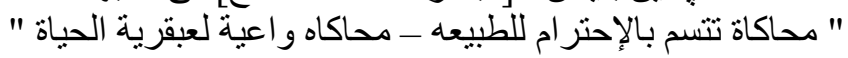

"A Respectful Imitation - A Conscious Emulation of life's genius"

::: Biomimicry - Biomimetics - Bionics - Bioinspiration :::

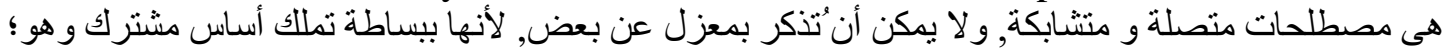

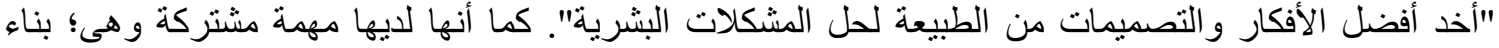

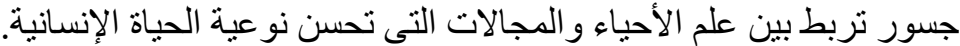

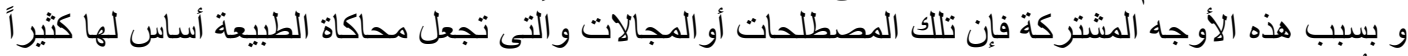

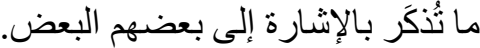

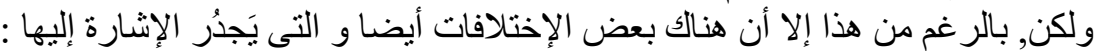
• •

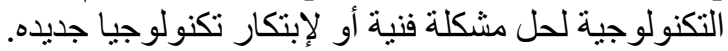

[Biomimicry]

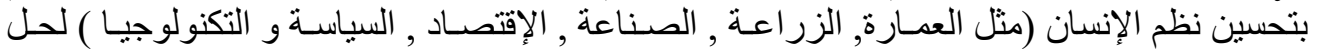

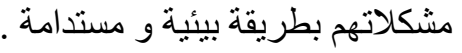

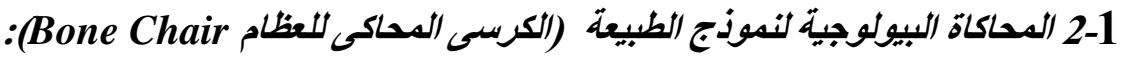

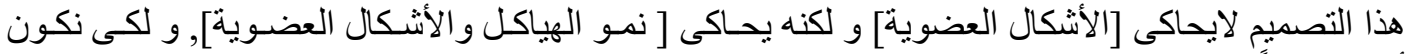
أكثر تحديداً فهو يحاكى [ الطريقة الفعاله لنمو العظام و المبادى التهـ التصميمية التى تقوم عليهـا تلك العمليـة].
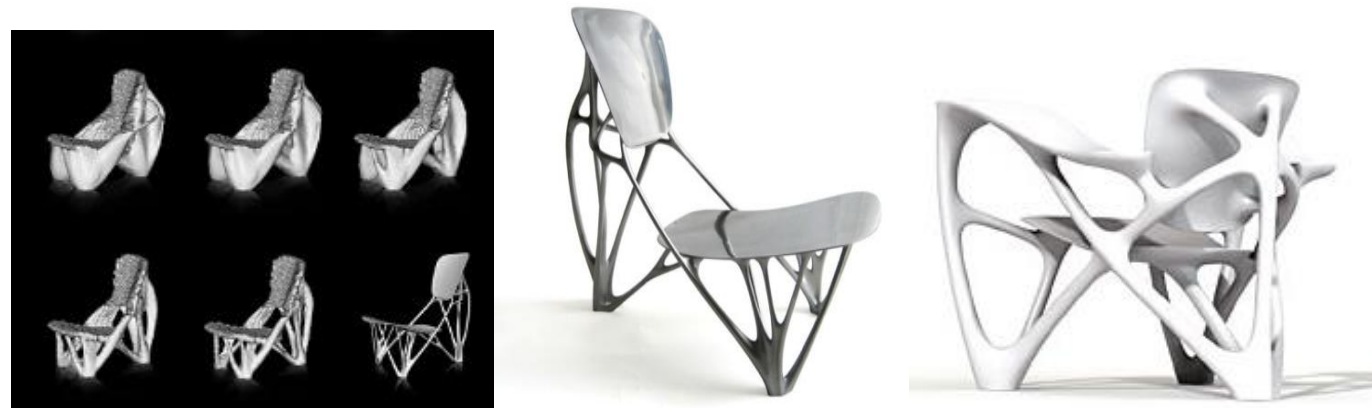

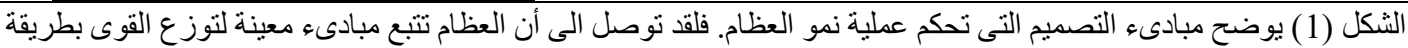

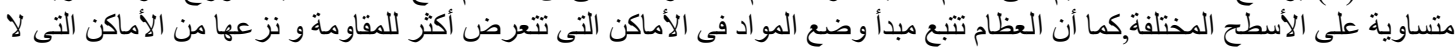
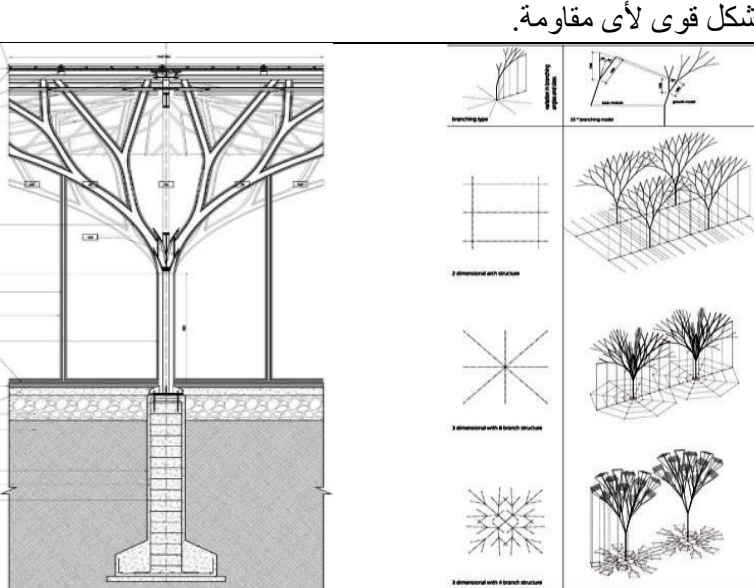

应说
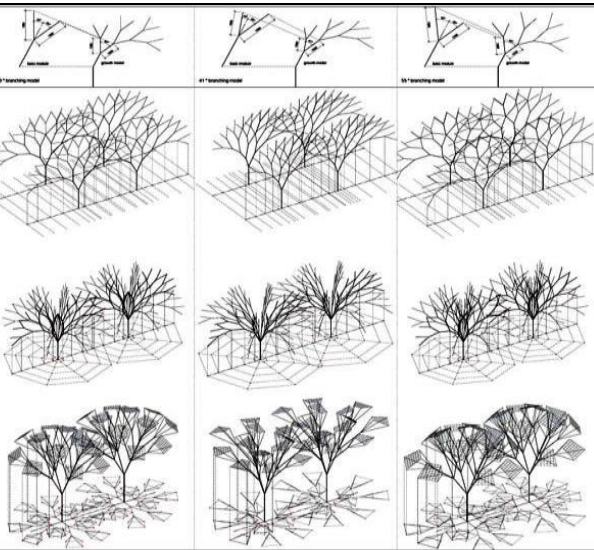


\section{INTERNATIONAL JOURNAL OF

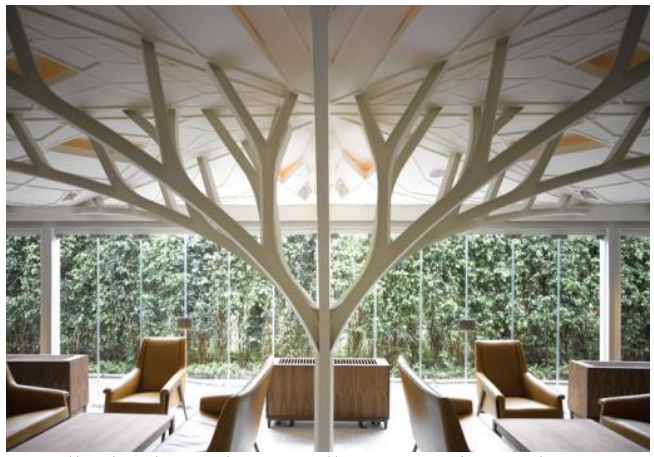

الثكل (2) يوضح مر احل بناء الثكل المتفرع، و التتفاصيل الانثائية للعمود و النظام الهيكلي للتصميم و الذي يتبع نظام نمو الأشجار المطيرة

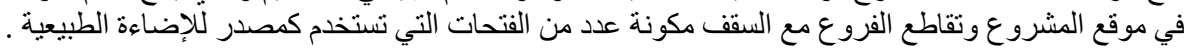

\section{3-1 المحاكاة البيولوجية لنموذج التكيف مع الطبيعة ( مواد تستقطب الماء ):}

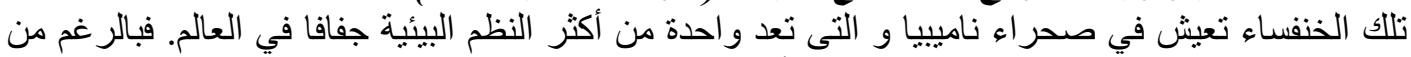

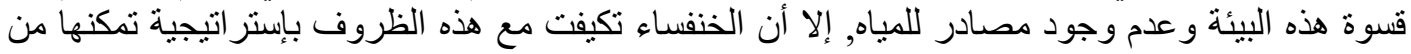

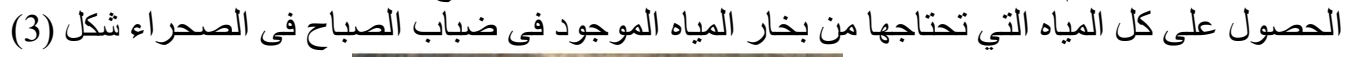

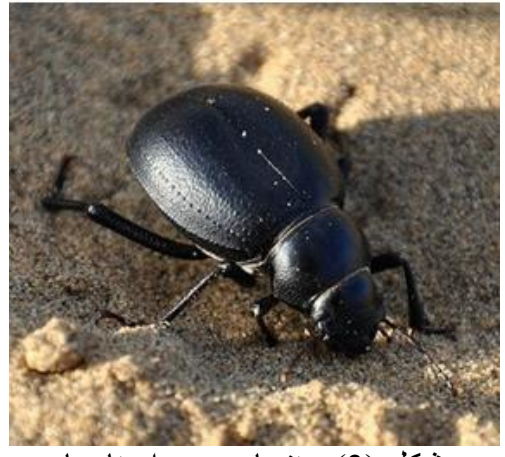

$$
\text { شكل (3) خنفساء صحر اء نامبيا }
$$

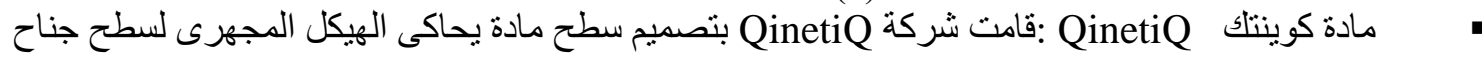

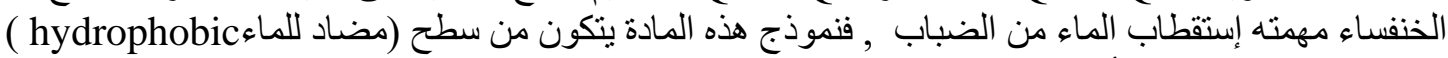

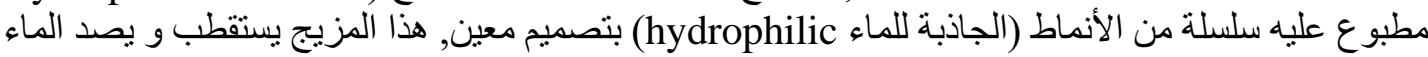

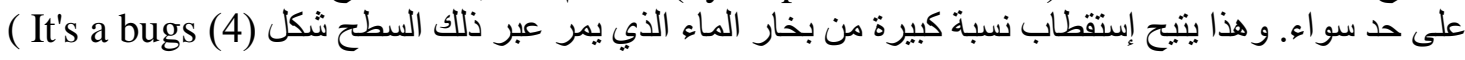

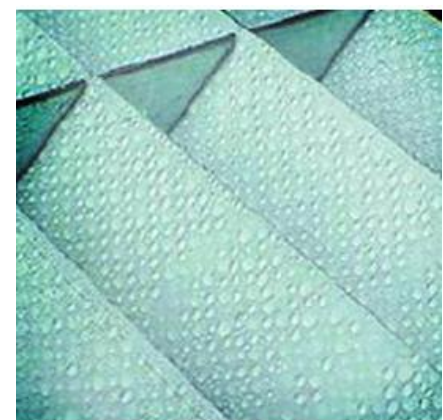

life, 2005)

شكل (4) وذللك بإتخاذ الخنفساء وضعاً معينً بحيث تجعل ظهر ها بمواجة الرياح المشبعة ببخار الماء, حيث يلتصق على سطحها هذا البخار

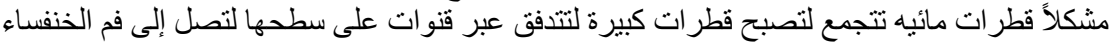




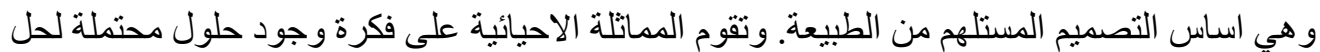

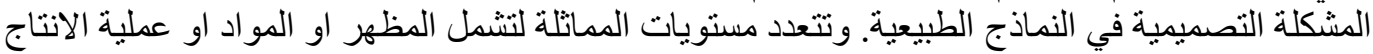

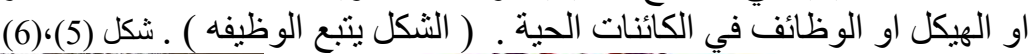

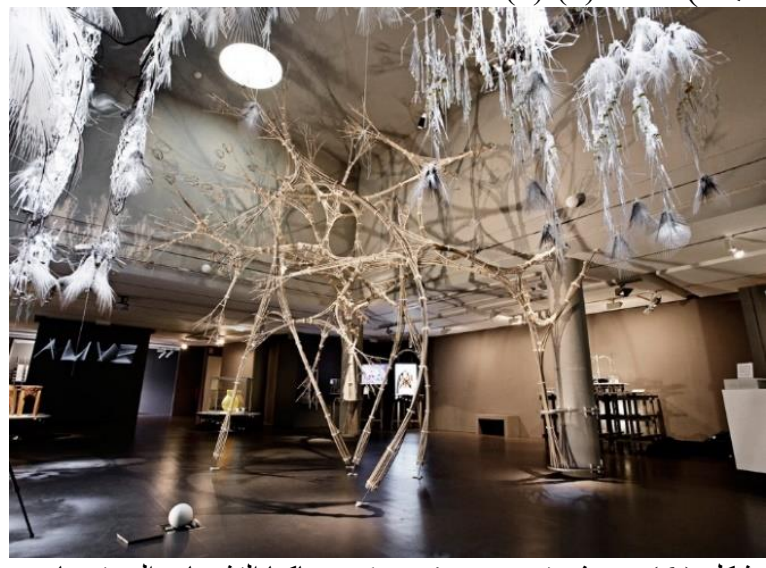

شكل ( 6): نموذج تصميم The Rise محاكيا التشعبات المستوحاة من الجهاز الدوري.

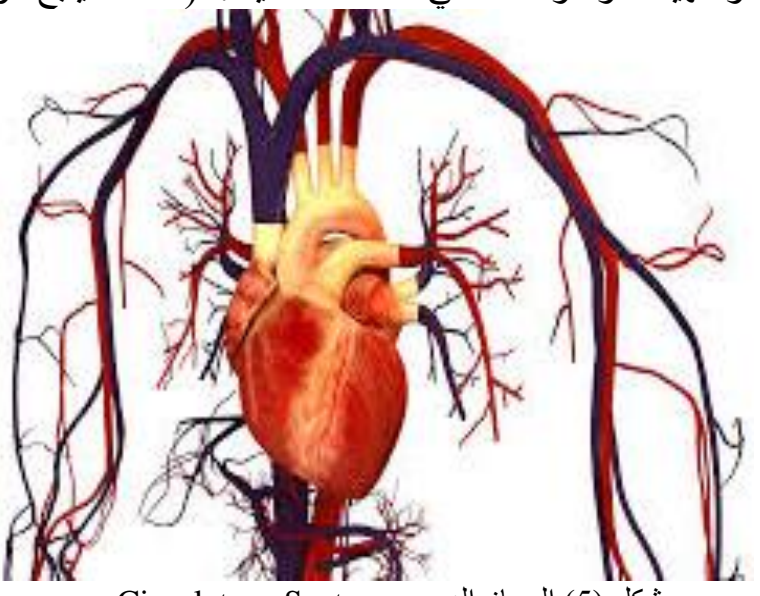

شكل (5) الجهاز الدوري Circulatory System

5-1 5-1 انماط المعاثلة البيولوجية:

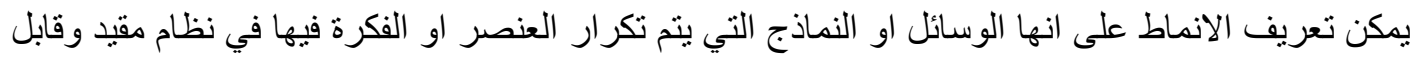

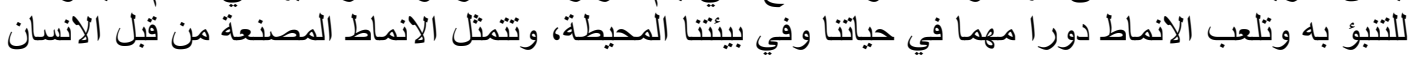

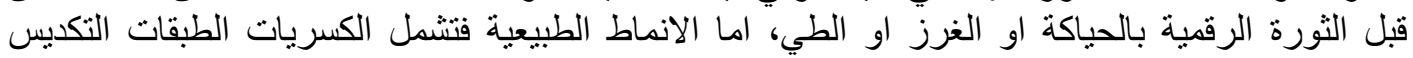

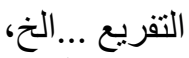
وتشهد العمارة المعاصرة تحول نحو الانماط الطبيعية بعد النطور ات التي شهدنها التكنولوجيا الرقمية لإنتاج

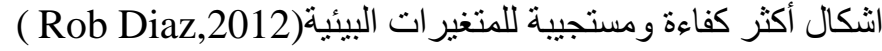

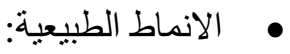

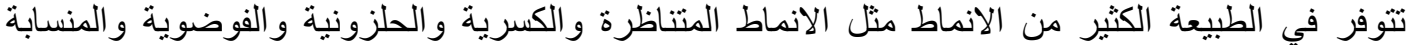

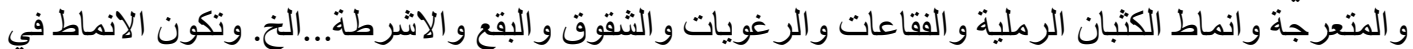

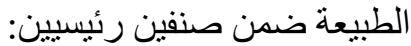

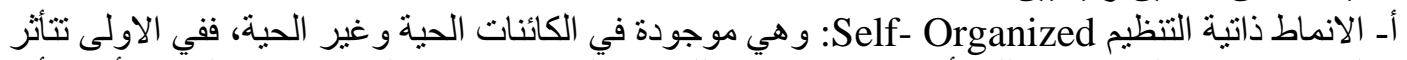

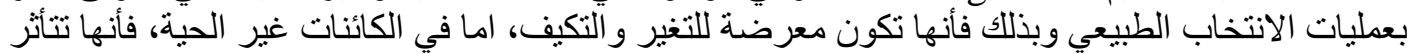

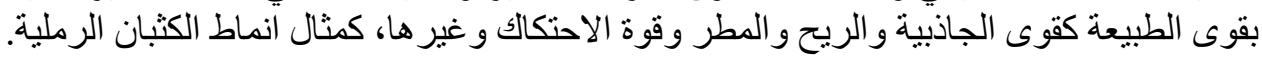

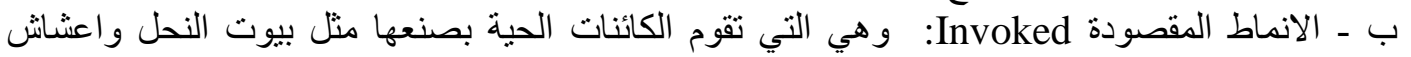

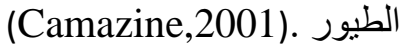
6-1 مستويات المماثلة البيولوجية :

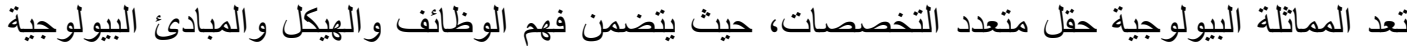

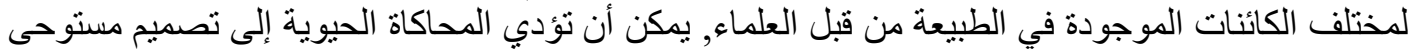

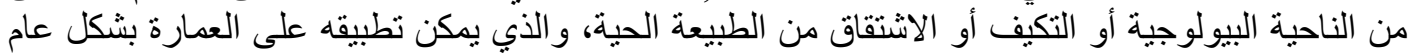

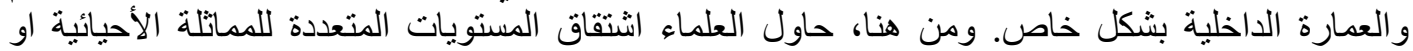

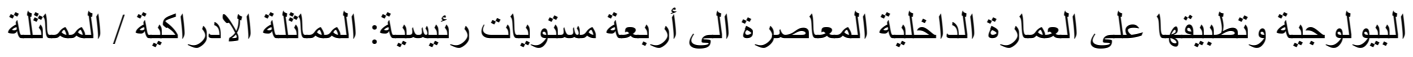

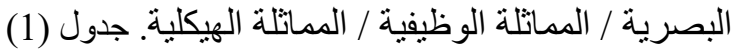
جدول (1) يوضح مستويات الممانلة البيولوجية.
رابعا
ثالثا
ثانيا 


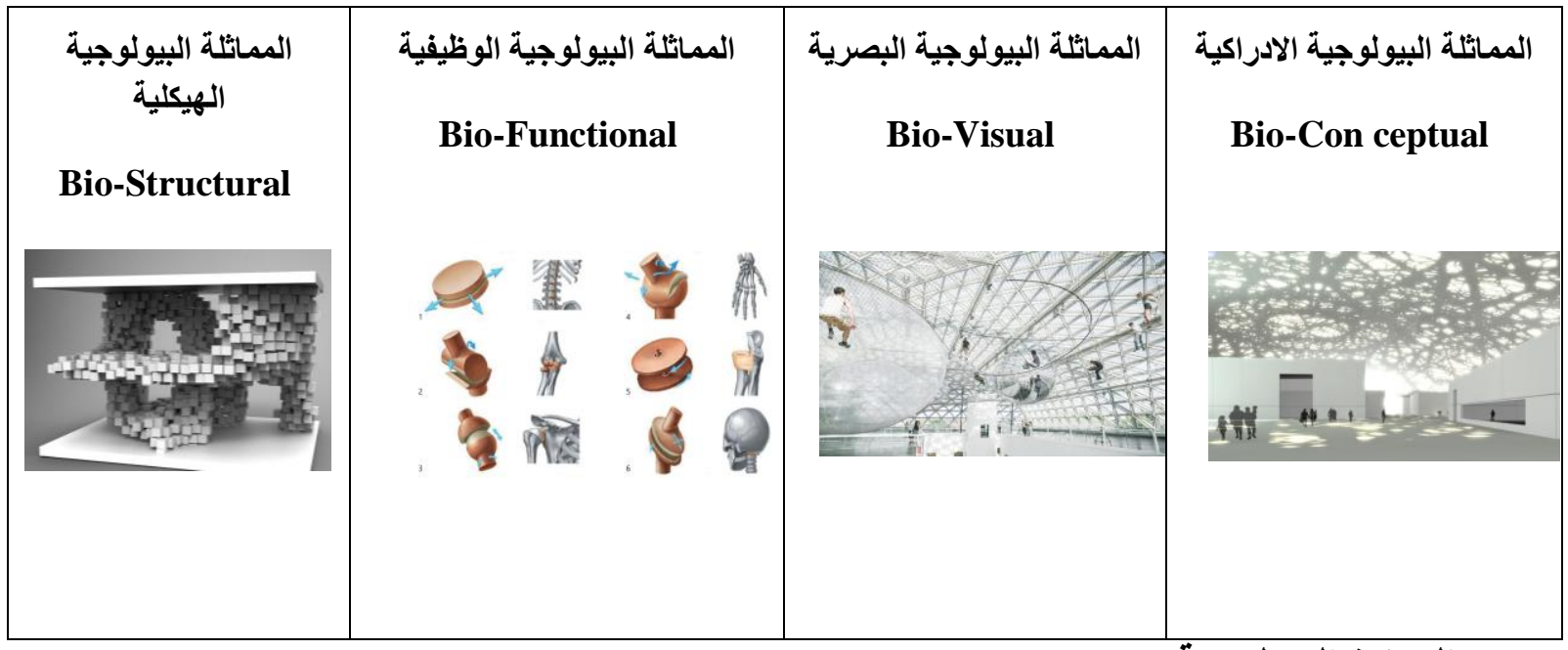

2- العمارة البيولوجية Bio-Architecture

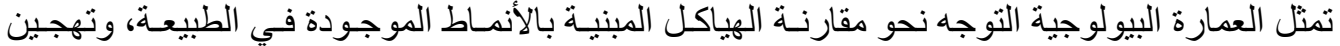

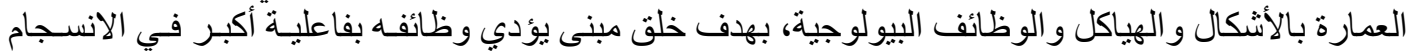

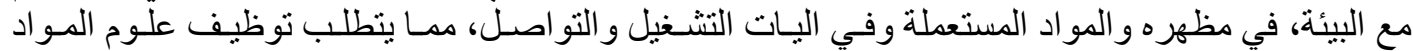

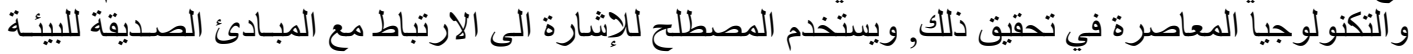

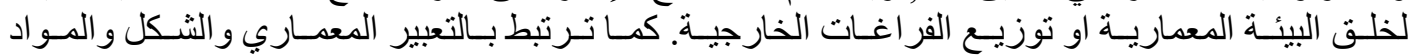

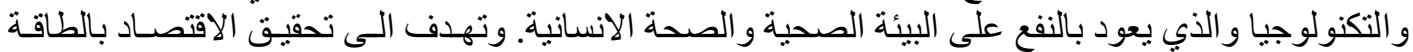

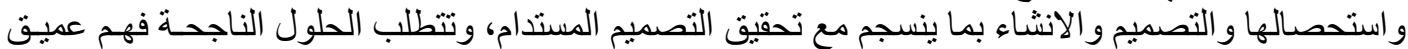

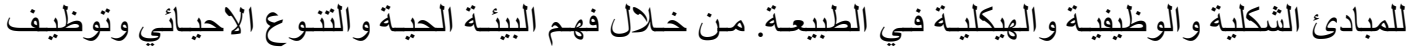
التكنولوجيا الصديقة للبيئة( Pourjafar,2011 )

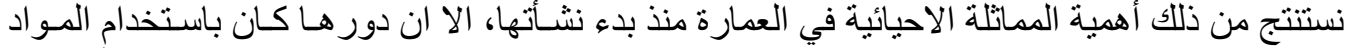

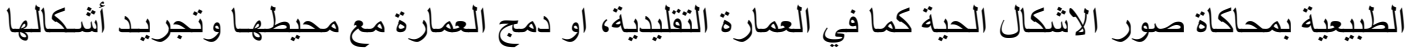

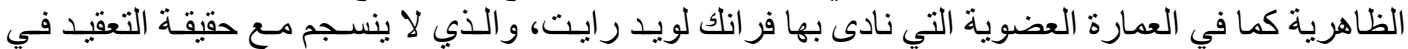

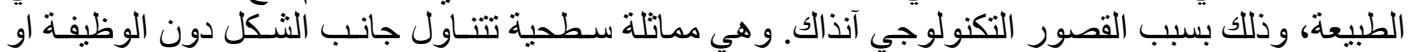

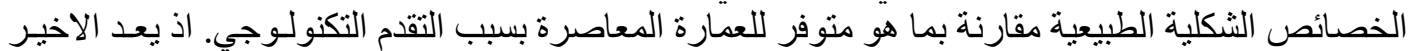

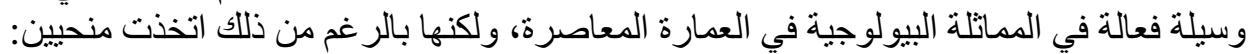

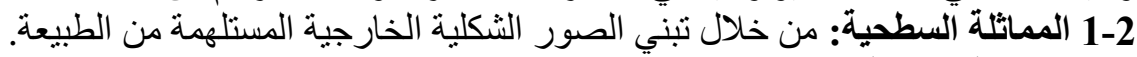

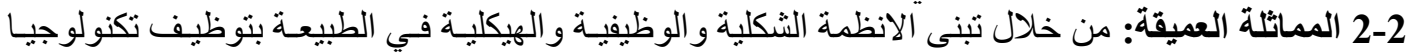

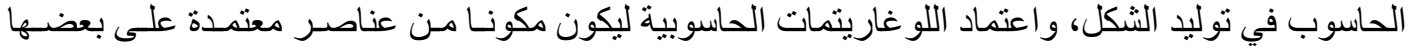

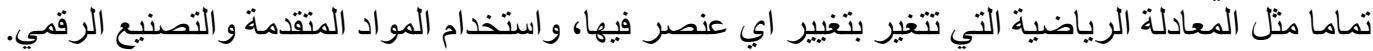

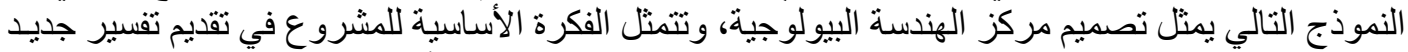

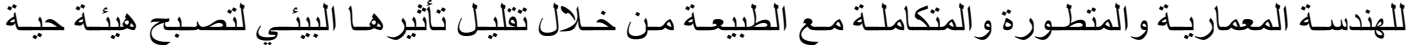

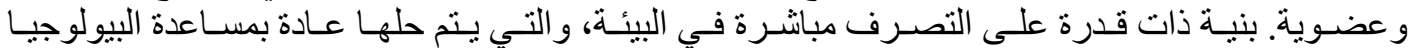

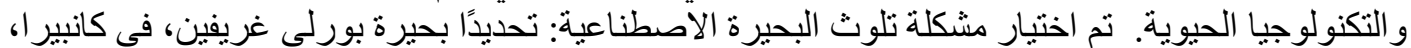

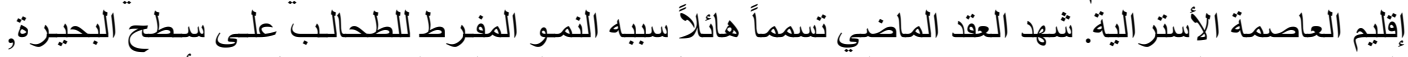

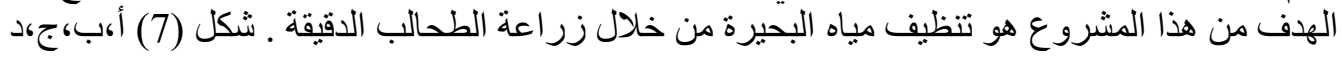

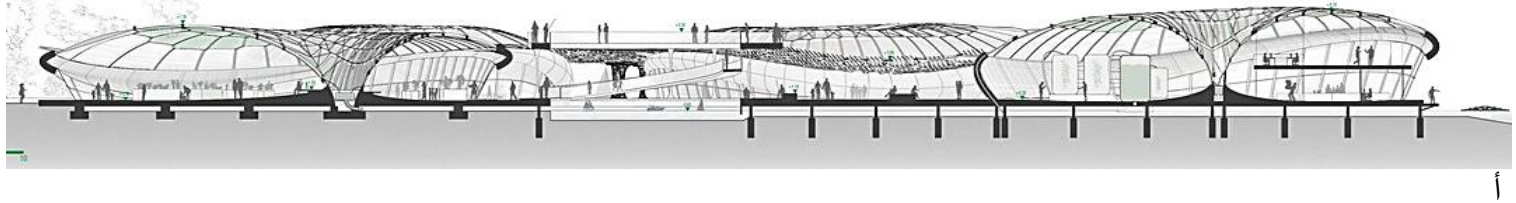



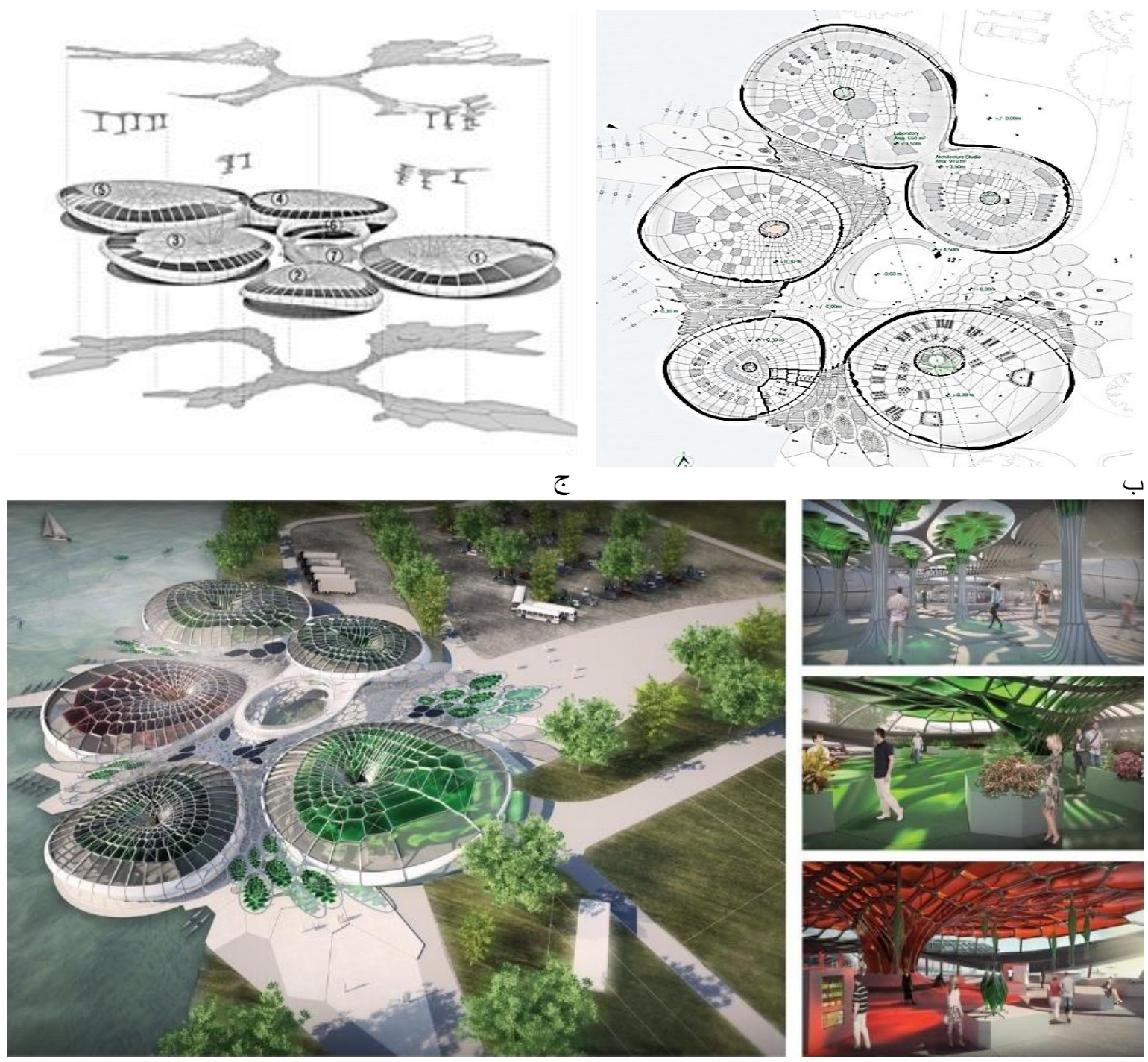

شكل (7) أكب،ج،د: تصميم مركز الهندسة البيولوجية الناتج من تفريعات انقسامات الخلايا المكونة لتشكيل المسقط الأفقي Bio ) Architecture Center | Andrea Dal Negro)

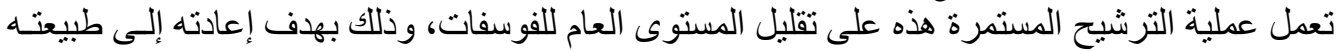

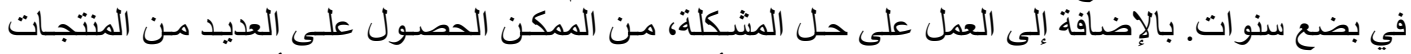

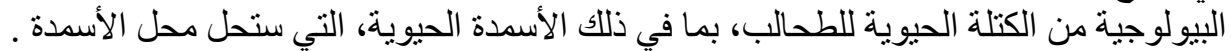

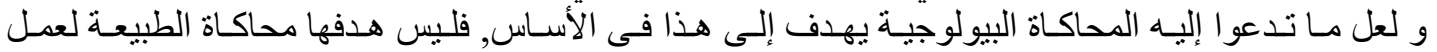

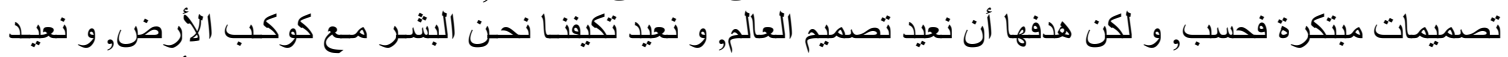

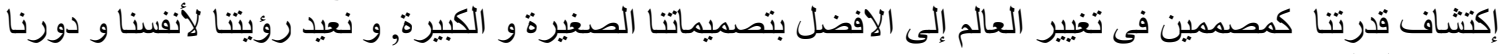

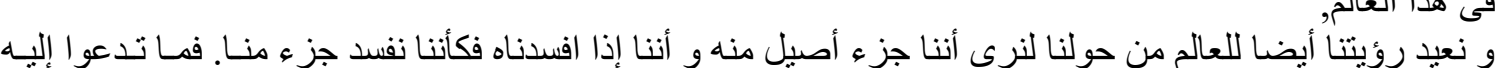

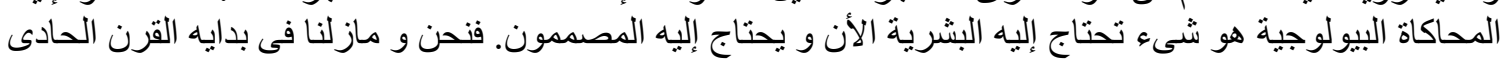

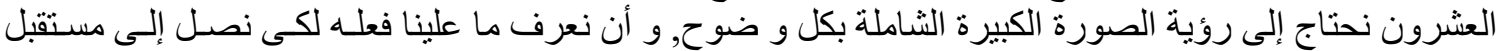




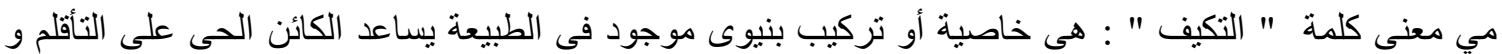

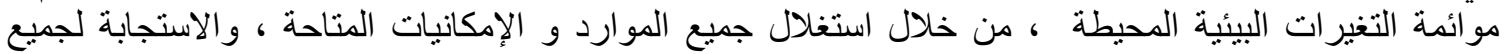
التغير ات ات اتغرة و و المؤثرات ، كما أنها تتصف بزيادة القدرة على البقاء .

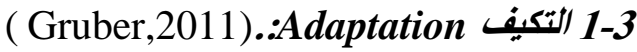

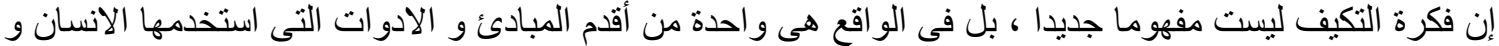

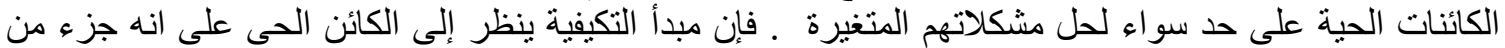

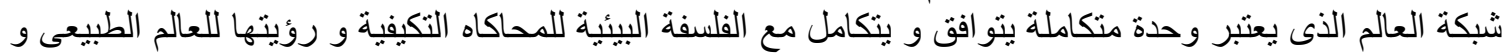

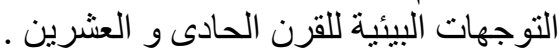

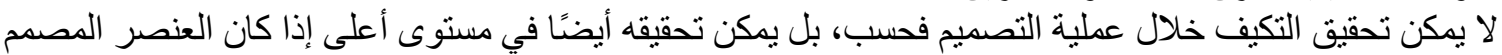
قادرًا ديناميكيًا على الاستشعار و الاستجابة لبيئة متغيرة. ينقسم التكيف تبعا للانظمة في في الطبيعة الى ثلاثة أقسام القام رئيسية:كما هو موضح بديجر ام 2 ، جدول 2.

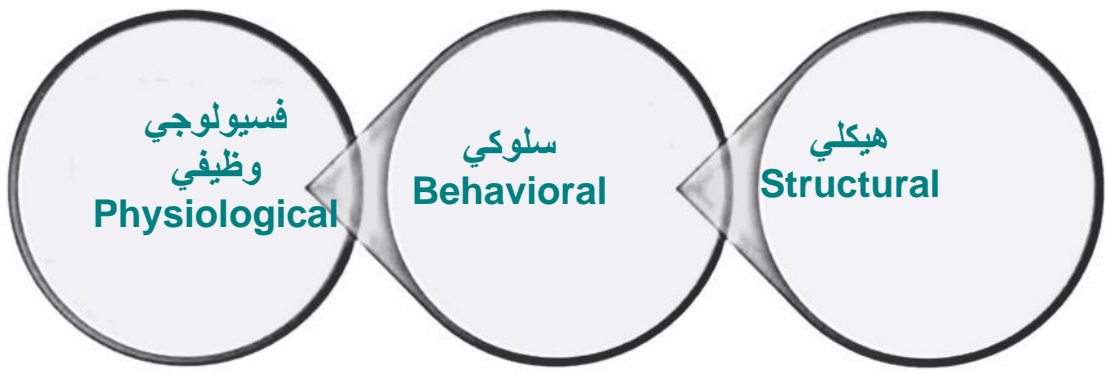

دايجرام (2): يوضح معايير التكيف تبعا للأنظمة التكيفية في الطبيعة

جدول(2) يوضح التكيف تبعا للأنظمة في الطبيعة

\begin{tabular}{|c|c|c|c|}
\hline الأنظمة الفسيولوجية الوظيفية & الأنظمة السلوكية & الأنظمة الهيكلية & المقارنة \\
\hline بأداء تفاعلات تعمل في الكائن الحي لتسيائية حيو ية محددة له & أساليب خاصة يقوم بها كائن حي للبقاء في بيئه الطبيعية & أجزاء أبهاء على قيد الحياة في بيئته الطبي تساعده على. & الطبيعة \\
\hline 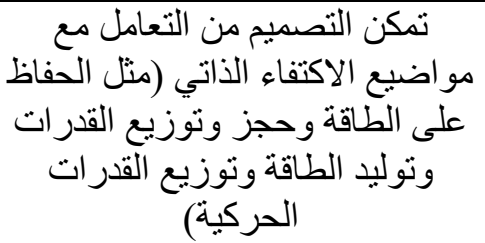 & 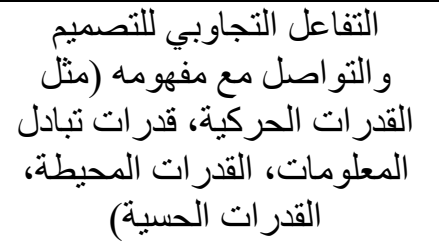 & 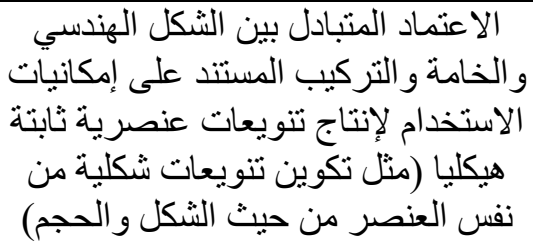 & التصميم \\
\hline
\end{tabular}

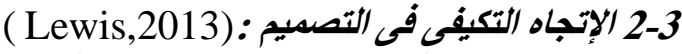

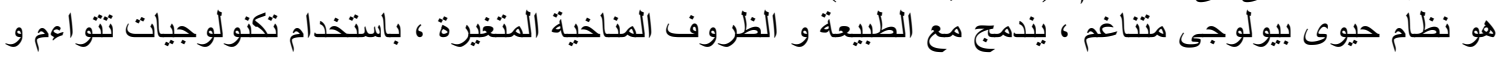

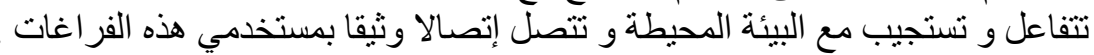

\section{3-3 تعريف مصطلح العدارة التكيفية :}

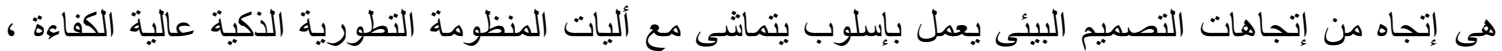

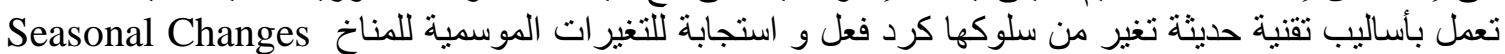
سواء كانت محلية أو عالمية ، مما ينتج عنها معايير تصميمية ذو منظومة المكة تكيفية للعمارة الذاخلية ، تتميز بالتكامل و

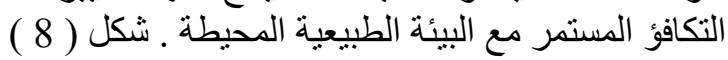




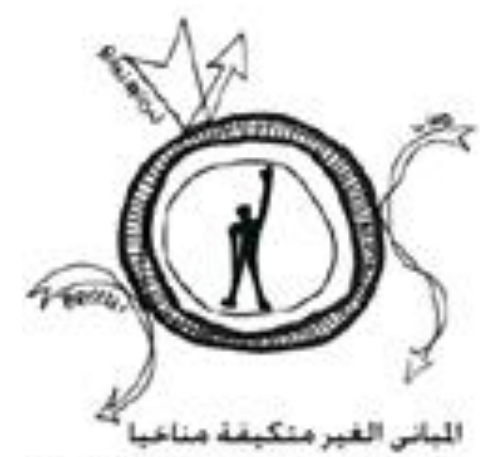

Cilmate rejecting buldings

$\downarrow$

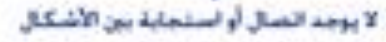

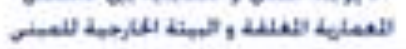

Vs

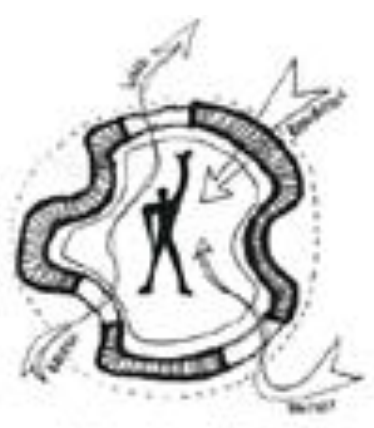

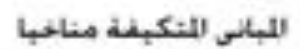

Climate adapfed buildings $\downarrow$

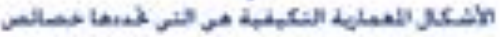

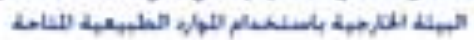

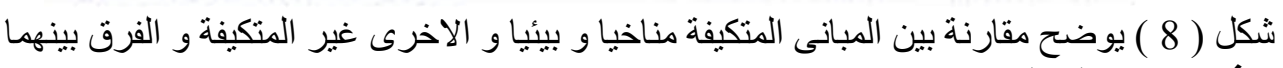

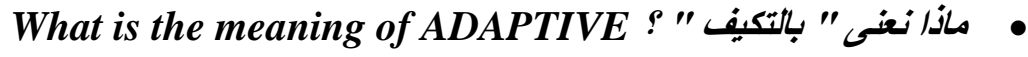

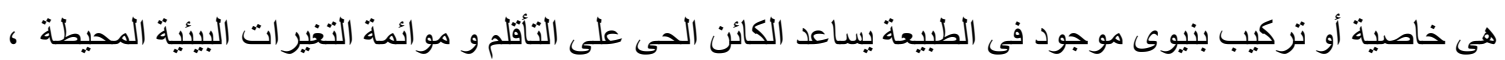

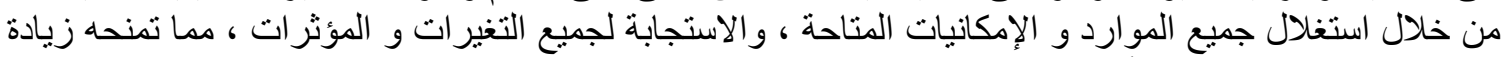

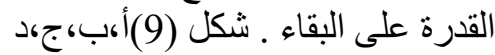

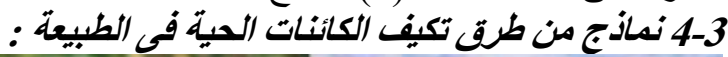

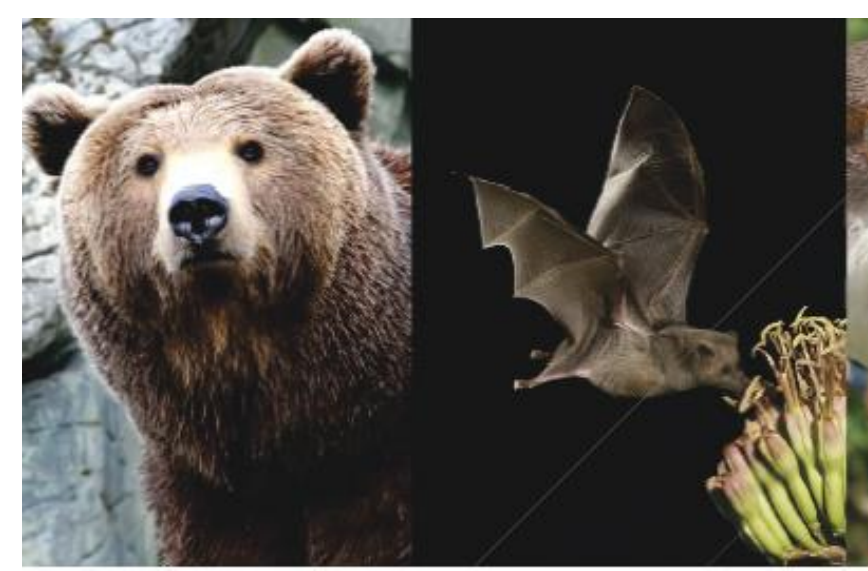

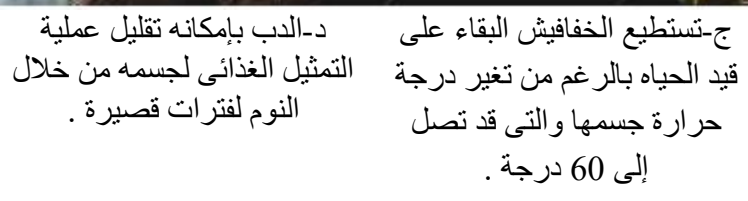
شكل (9) أ،ب،جهد نماذج من طرق تكيف الكائنات الحية فى الطبيعة.

5-3 عوامل ظهور العمارة التكيفية :

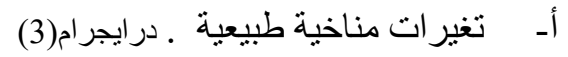
ب- ت تغير ات مناخية سببها الإنسان . 


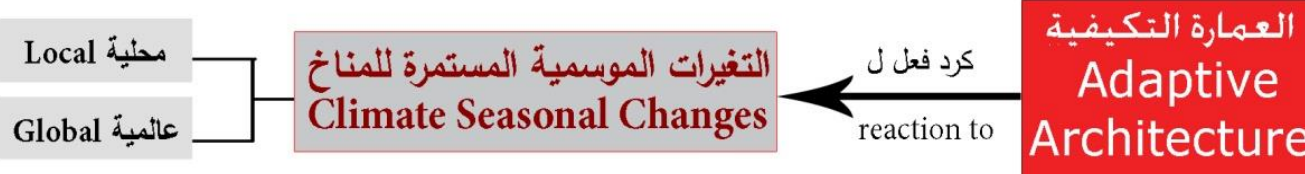

الفراغات الإخلية Interior

Architectur

$$
\text { در ايجر ام(3) يوضح التغيرات الموسمية للمناخ كأحد أهم عوامل ظهور العمارة التكيفية. }
$$

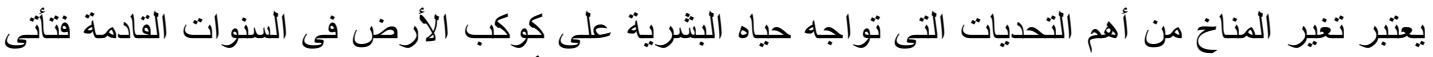

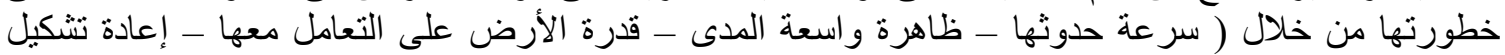

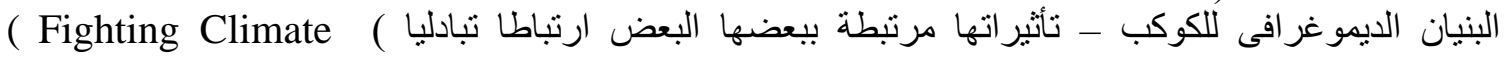

Change: Human Solidarity in a Divided World,2007-2008)

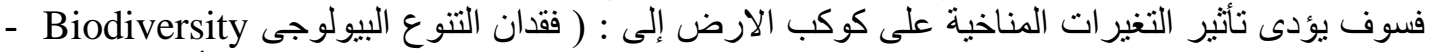

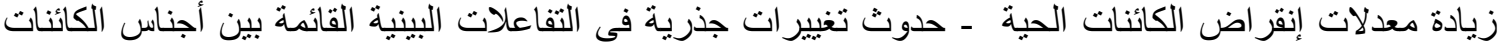

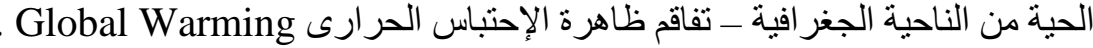

6-3 "التكيف و التخفيف" ودور هم فح كيفية التغلب على مشكلة التغيرات المناخية المستمرة:

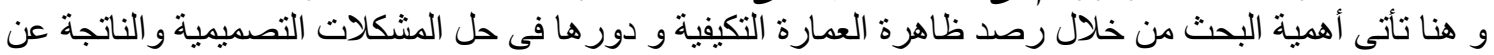

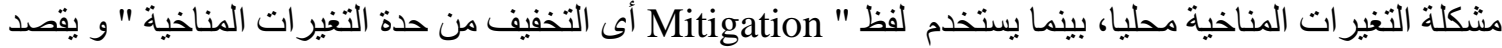

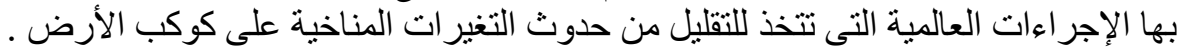

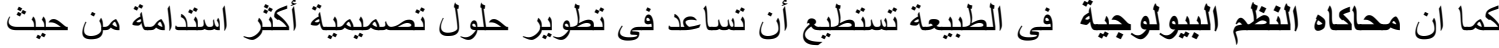

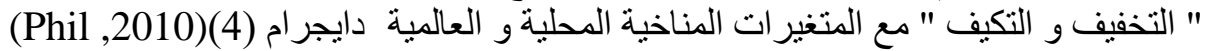

التخفيف من ظاهرة الإحتباس الحرارى من طرى

Mitigation يقصد به : الإجراءات العالمية

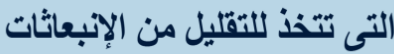

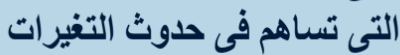

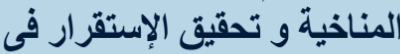

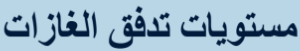

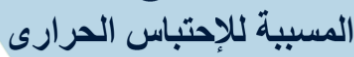
فى الغلاف الجوى.
الطاقة الحرارية: الأرضية Geothermal :الطاقة الثمسية: Solar Thermal |التففئة المركزية District Heating * التهوية الطبيعية: Natural Ventilation *زراعة الأسطح Green Roofs * المحافظة طلى المياه Water Conservation
التكيف مع ظاهرة الإحتباس الحرارى

\section{Adaptation}

يقصد به : الإجراعات المحلية التى تتخذ لتقليل أو منع حدوث الأثثار

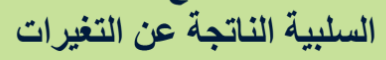
المناخية و كيفية التأقلم معها التفرات الفية سواء الفعلية الحالية أو المتوقع حوثها فى المستقبل .

دايجر ام (4) يوضح كيفية التغلب على مشكلة التغيرات المناخية المستمرة من خلال التكيف و التخفيف من حدوثها ، وما هى استر اتيجيات تطبيقهم على الفر اغات الداخلية.

العمارة التكيفية و استراتيجيات تصميم الفراغات البيئية :

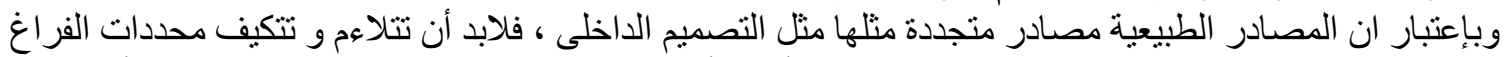

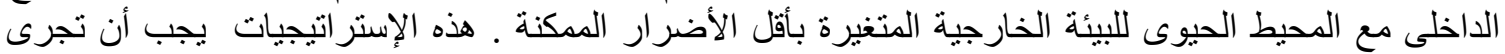
بشكل لانهائى على مدى أجيال عديدة من خلال تلتع مسار تكيف الكائنة الكائنات الحية من خلال :

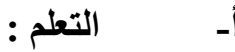
من الطبيعة كيف تتم عملية التكيف و استنباط الافكار و الطرق البيولوجية البيئية لحل التحديات التكنولوجية فى

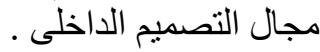

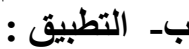




\section{INTERNATIONAL JOURNAL OF \\ DESIGN AND FASHION STUDIES}

VOLUME 4, ISSUE 1, 2021, 22-38.

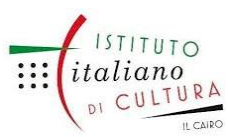

www.egyptfuture.org/ojs/

على التصميم المادى ( كالهياكل المعمارية و النظم التكيفية للفر اغات الداخلية ) ، كما انها يمكن ان تطبق على التى التئ

التصميم غير المادى ( كبر امج الحاسب الالى و المعارية الانظمة التكنولوجية ).

تيـ الامج : بلنيع الطبية و النظم البشرية باستخدام احدث الوسائل التكنولوجية لتحسين البيئة المبنية

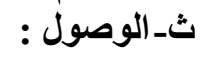

إلى استر اتيجية تكيفية واعية من قبل المصممين لمو اجهة تحديات و مشاكل التصميم على نحو اكثر تفاعلية وديناميكية و إستنامة .

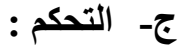

التحكم البيئى التكيفى للكتلة الفر اغية ـ شكل(10)
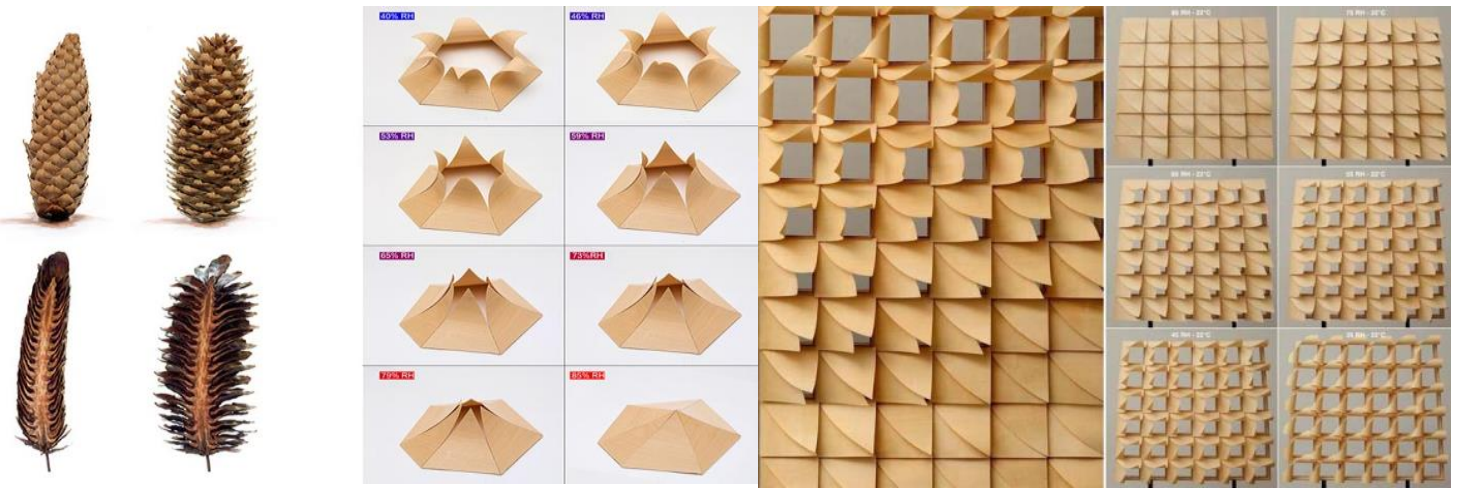

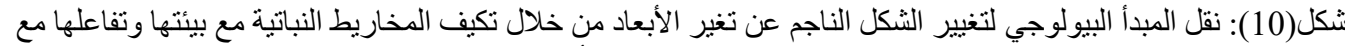

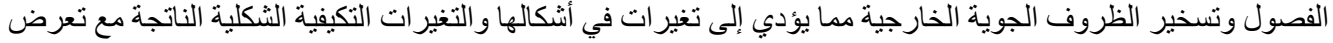

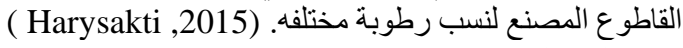

: 7-3 خصائص فكر التصميم التكيفيى

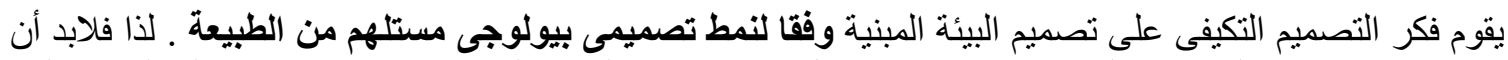

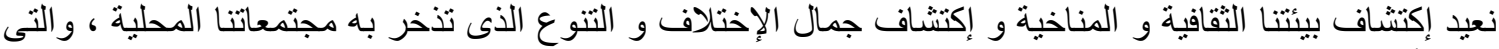

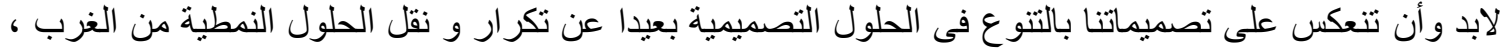
وذللك لأن كل بيئة طبيعة لديها حلول و إستر اتيجيات خاصة و متنو عة فى التكيف و البقاء ـ شكل (11) 


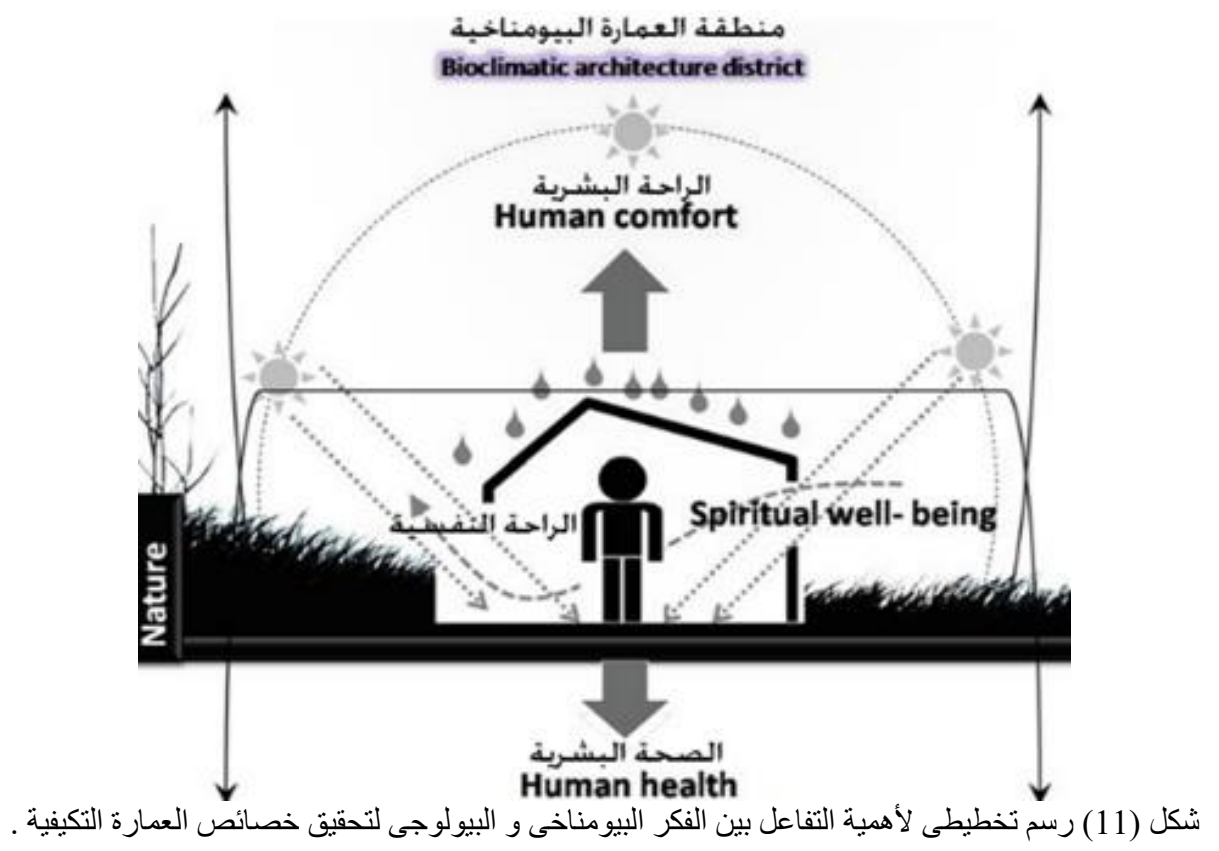

فمن أهم خصائص الفراغات الداخلية التكيفية :هو التفاعل بين الفكر البيومناخى و البيولوجى فى تصميم الفر اغات الداخلية

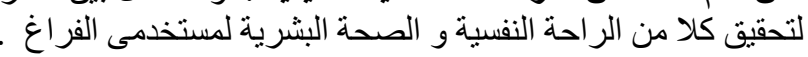

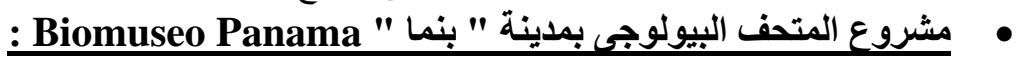

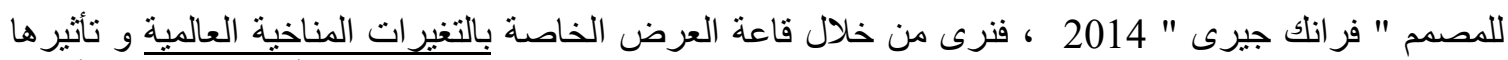

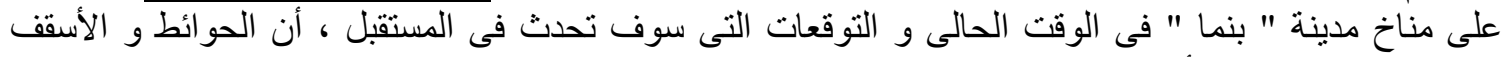

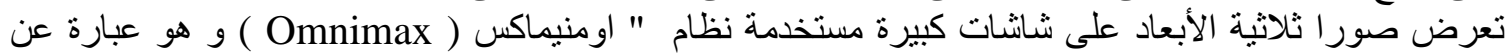

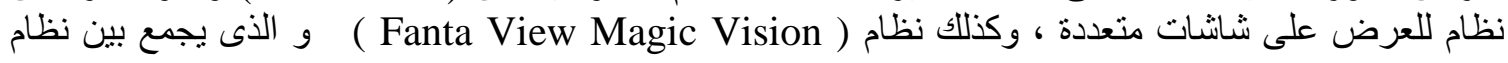
العرض الضوئى للصورة مع التعبيرات التشكيلية ـ شكل(12)،(13)(Biomuseo,2021)
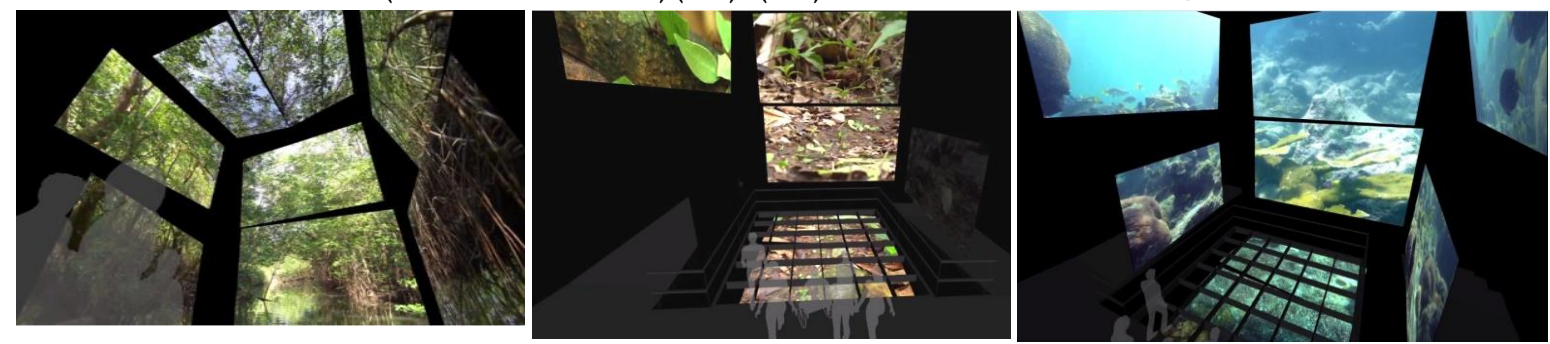

شكل(12) اللقطات المنظورية البانور امية لقاعة العرض الخاصة بالتغير ات المناخية المزودة بتكنولوجيا الأسقاط الضوئى فى المتحف

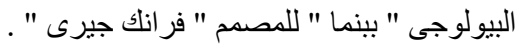




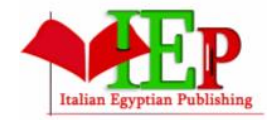

ISSN: $2735-5365$

\section{INTERNATIONAL JOURNAL OF \\ DESIGN AND FASHION STUDIES}

VOLUME 4, ISSUE $1,2021,22-38$. $\overline{\vdots: \vdots:\left(\begin{array}{c}\text { ISTITUTO } \\ \text { italiano } \\ \text { DI CULTURA }\end{array}\right.}$

www.egyptfuture.org/ojs/
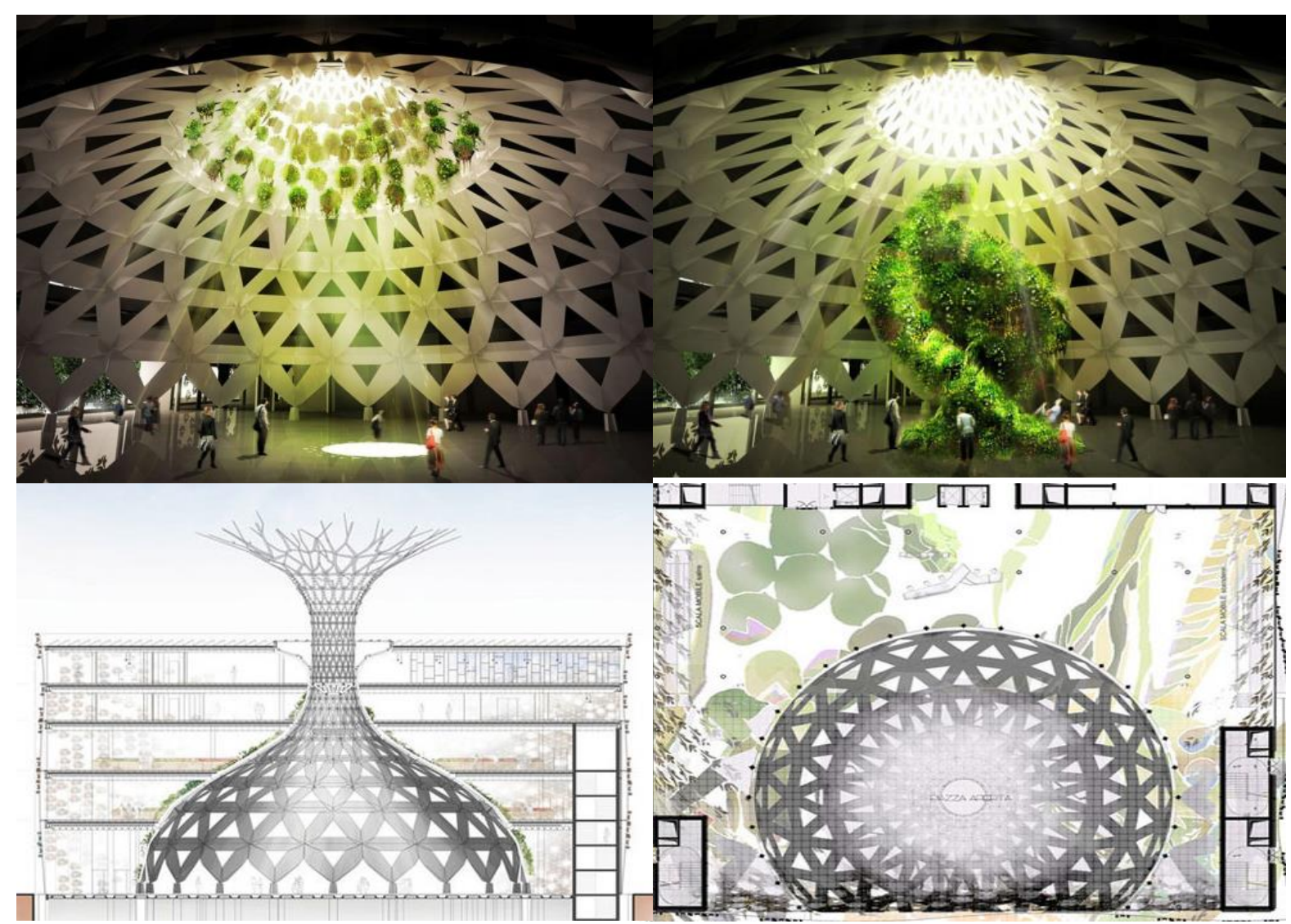

شكل (13) المسقط الأفقى والقطاع الراسى المستوحى من البيئه وسائل العرض التكيفية لديها قدرة عالية على خلق أجواء

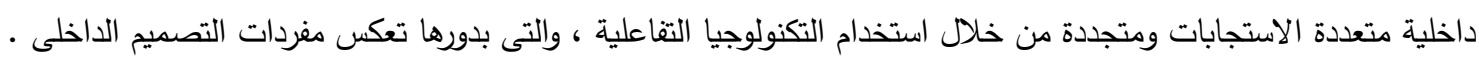

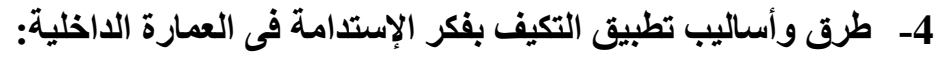

حدد الاتحاد الدولي الحفاظ على طبيعة التنمية المستدامة من حيث صلتها بجمئ الإسئ جوانب الإستدامة و التى تسعى

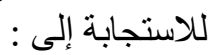

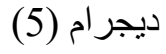

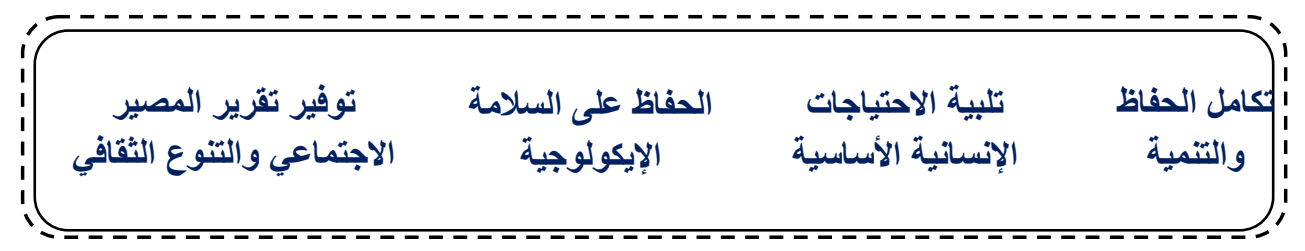

ديجر ام (5) أهداف التنمية المستدامة التكيفية

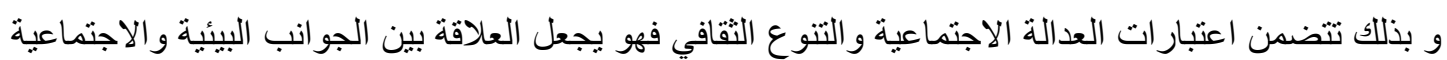

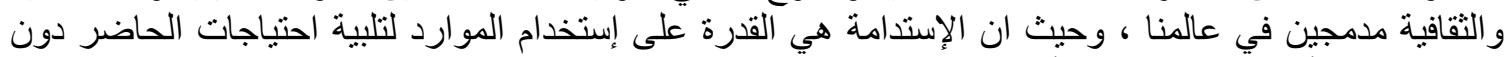

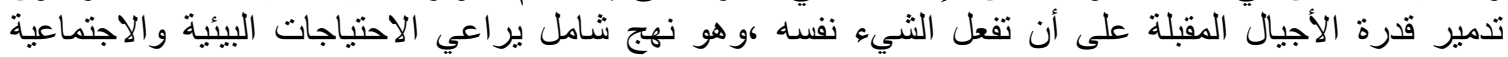

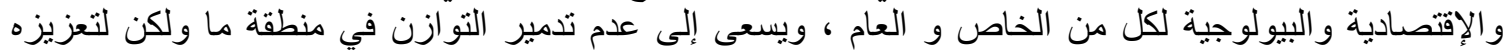

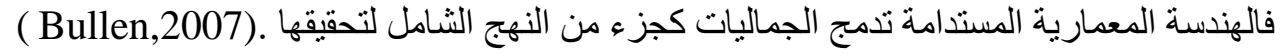




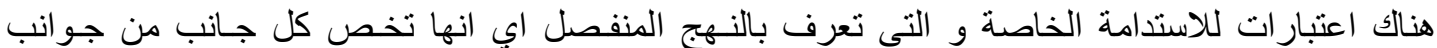

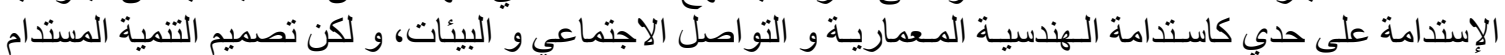

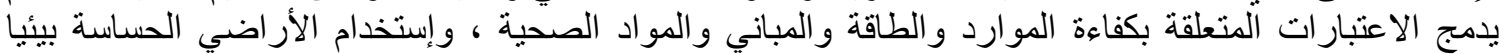

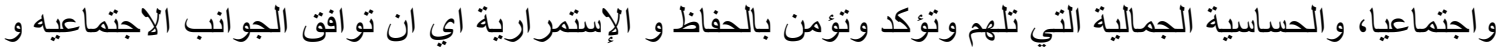

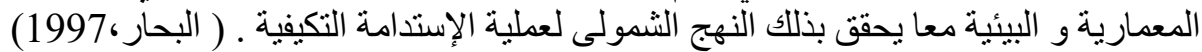

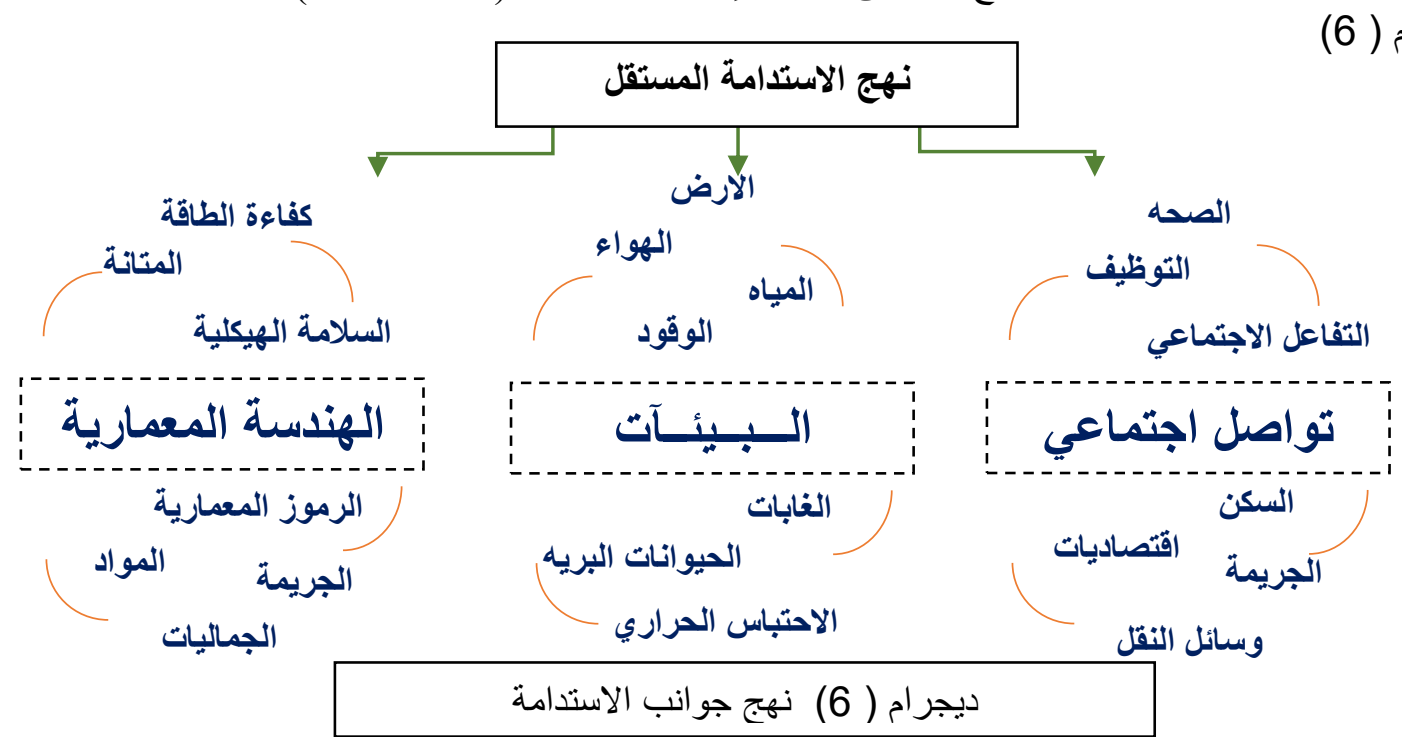

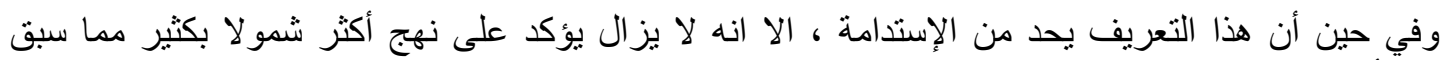

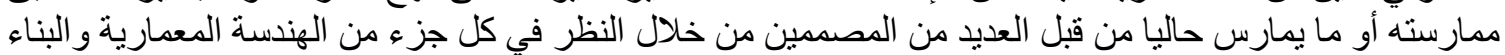

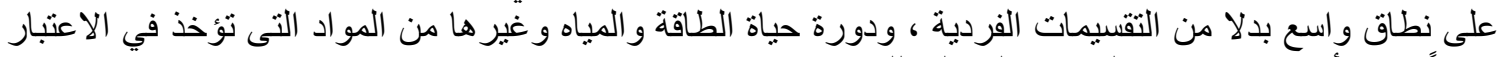

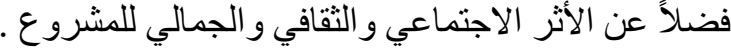

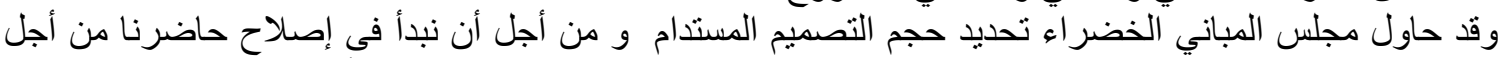

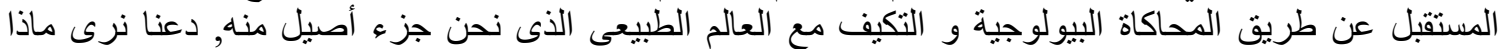

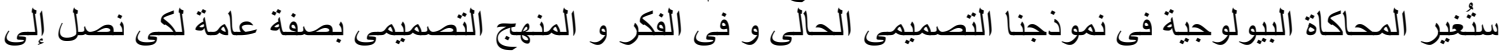

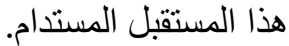
توضيح النهج الثمولى للجو انب النى تحقق الاستدامة. ديجرام ( 7)

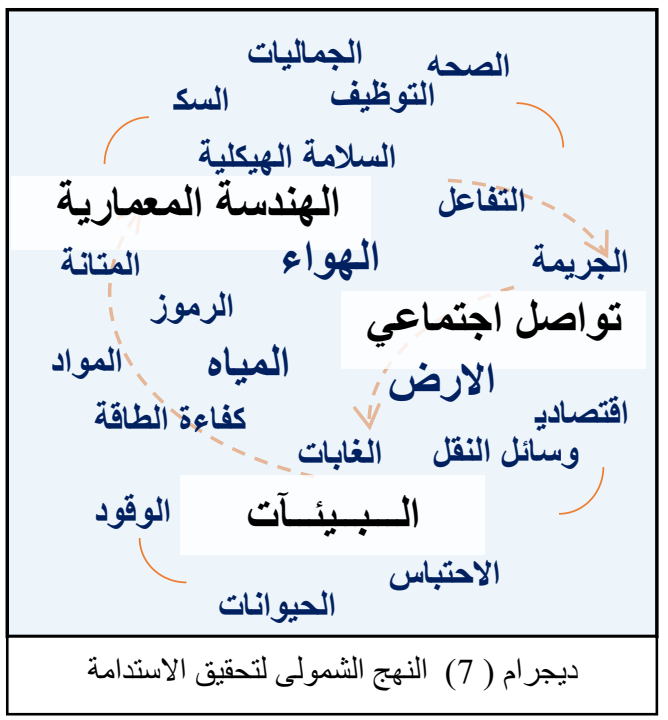




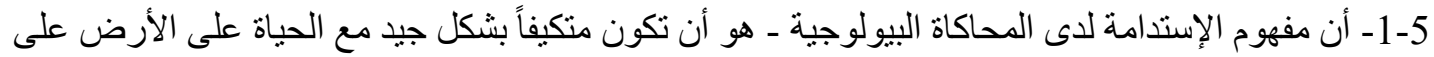

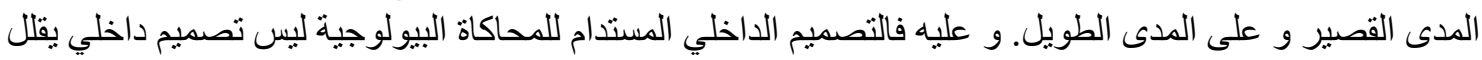

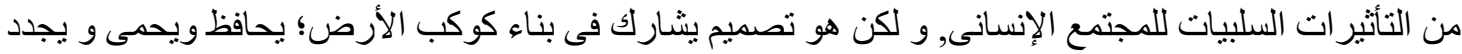
الأنظمة البيئية و المجتمعات الإنسانية و الطبيعية.

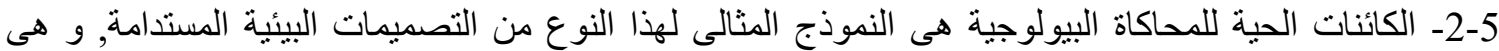

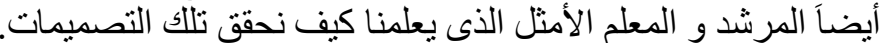

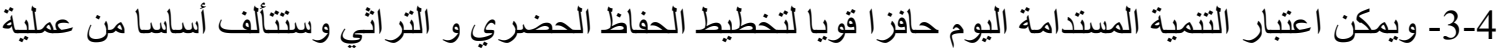

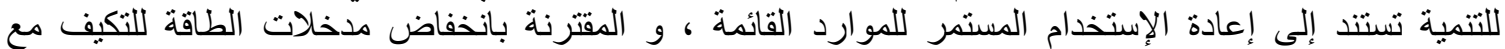

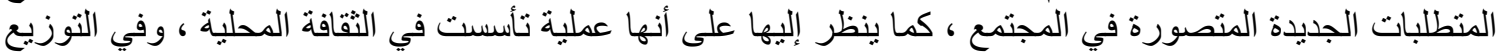

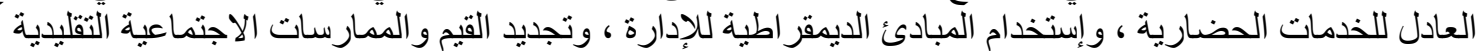

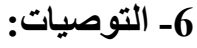

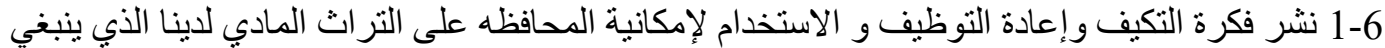

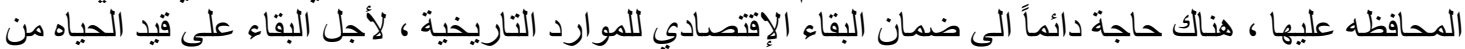

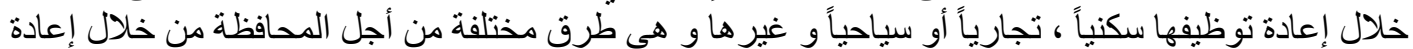

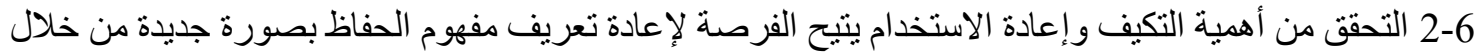

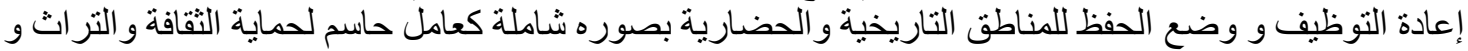
نو عية البيئة الطبيعية و المبنية للأجيال القادمة للأستمتاع بها ، و أهمية الحفاظ على ما لديهم من ثروة حقيقية تستحق

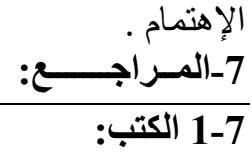

1. Diaz, Rob Diaz, 2012, "Intelligent Façade Engineered through MorphoEcogenetic Aggregates", Ms. Thesis, Texas Tech University, P. 88.

2. Harysakti ,Ave. Biophilic and Bioclimatic Architecture - Analytical Therapy for the Next Generation of Passive Sustainable Architecture, Amjad Almusaed,Springer ,2015

1. Pourjafar, Mohamad Reza; Mahmoudinejad, Hadi \& Ahadian, Omid, 2011, " Design with Nature in Bio-Architecture Whith emphasis on the Hidden Rules of Natural Organism", International Journal of Applied Science and Technology Vol. 1 No.4; July 2011, p.79

2. Zerebakova, Petra; Šindelář, Jiří; Kuchta, Ladislav \& Repka, Matúš, 2007, "

3. Survey in the Field of Bio-Architecture", EU Community Initiative INTERREG III B 5)-CADSES, Neighbourhood Program, p.5, 14.

4. Human Development Report - Fighting Climate Change: Human Solidarity in a Divided World, Chapter1, 2007/2008 , p:21

5. Phil, Young Lit, Environment \& Systems Integration, OJSturdyArchitecture ,Diver , 2010.

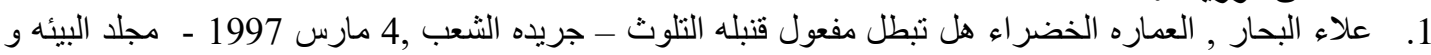

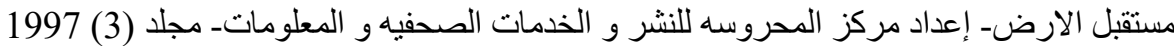

2. "It's a bugs life" Building services journal. 06 Jan (2005) http://www.inventapartners.ltd.uk/images_folder/Inventa\%20Jan05.pdf 
3. Montana, Carlos. - An Interview with Biomimicry Guild Principals Janine Benyus and Dayna Baumeister.FuturArc magazine. 1st Quarter (2009) (http://www.futurarc.com/previous edition/table of contents 1Q 2009.cfm)

4. Benyus, Janine M. "Biomimicry: Innovation Inspired by Nature" Harper Perennial (1997)

5. "What is Biomimetics?" A radio interview with Julian Vincent broadcast on New Zealand Public Radio Department of Mechanical Engineering, University of Bath .( http://www.bath.ac.uk/mech-eng/biomimetics/about/interview-nz.html)

6. Alberto M. H, Carlos. "Nature Informs Design: An Interview with Biomimicry Guild Principals Janine Benyus and Dayna Baumeister" FuturArc magazine. 1st Quarter (2009))-http://www.futurarc.com/previous_edition/janine.cfm

7. Camazine, Scott, "Self-organization in Biological Systems", Princeton University Press. p.vii,2001.

8. Gruber, , Biomimetics in Architecture, Springer Vienna, Vienna,2011

9. Lewis, Ramola Helena , Re-architecture_Adaptive Reuse of buildings - with focus on interiors , 2013

10. Bio Architecture Center | Andrea Dal Negro.( https://www.rethinkingthefuture.com/idsa2016-mixed-use/bio-architecture-center-andrea-dal-negro)

11. Human Development Report - Fighting Climate Change: Human Solidarity in a Divided World, Chapter1 , 2007/2008 , p:21

12. International Journal of Applied Science and Technology Vol. 1 No.4; July 2011, p.79

13. Bullen, Peter A. "Adaptive reuse and sustainability of commercial buildings." Facilities 25.1/2 (2007): 20-31.

1. 282001 عمارة مستدامة. 28 (مايو 2.

(.https://ar.wikipedia.org/wiki/\%D8\%B9\%D9\%85\%D8\%A7\%D8\%B1\%D8\%A9_\%D9\%85\%D 8\%B3\%D8\%AA\%D8\%AF\%D8\%A7\%D9\%85\%D8\%A9)

2. Glossary of sustainability terms .(21 May 2021).

(http://www.appropedia.org/Glossary_of_sustainability_terms)

3. http://www.bath.ac.uk/mech-eng/biomimetics/about/interview-nz.html

4. https://www.slideshare.net/sayedmarey/00-fin0al0

5. https://www.re-thinkingthefuture.com/idsa2016-mixed-use/bio-architecture-center-andreadal-negro

6. (Biomuseo), (http://www.biomuseopanama.org/en/meet-biomuseo)

7. Fog-catching material.

(http://asknature.org/product/ce46c846e11fb2e99eff7f1143df3bd3)

8. http://www.sustainablesarasota.com/ssDocuments/6/EarthDay2004.pdf 